\title{
Stille/Diels-Alder Reaction Sequences: Diversity-Oriented Access to Novel Steroids
}

\section{Hans Wolf Sünnemann, ${ }^{\dagger}$ Anja Hofmeister, ${ }^{\ddagger}$ Jörg Magull,, ${ }^{\ddagger}$ Martin G. Banwell" and Armin de Meijere ${ }^{\star, \dagger}$}

Institut für Organische und Biomolekulare Chemie and Institut für

Anorganische Chemie der Georg-August-Universität, Tammannstr. 2-4, 37077

Göttingen, Germany, and Research School of Chemistry, Institute of Advanced

Studies, The Australian National University, Canberra, ACT 0200, Australia

armin.demeijere@chemie.uni-goettingen.de

\section{Contents}

General Experimental

Representative Procedures for the Preparation of Selected Compounds

Spectral Data for Remaining Compounds

References

${ }^{1} \mathrm{H}$ and ${ }^{13} \mathrm{C}$ NMR Spectra of Compounds $\mathbf{3}$, cis-4, trans-4, trans-5, trans-6,

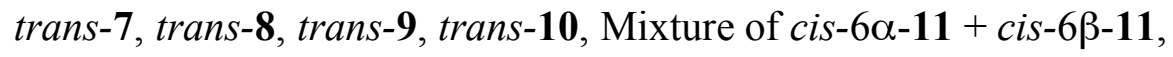

Mono-deprotected Derivative of trans-6, trans-12,

Mono-deprotected Derivative of trans-5, trans-13, trans-14, and 15

\footnotetext{
$†$ Institut für Organische und Biomolekulare Chemie der Georg-August-Universität

$\ddagger$ Institut für Anorganische Chemie der Georg-August-Universität

I The Australian National University
} 
General. ${ }^{1} \mathrm{H}$ NMR spectra were recorded on a Bruker AM 250 instrument operating at $250 \mathrm{MHz}$. Chemical shifts are reported as $\delta$ values relative to residual chloroform $(\delta=7.26)$ or benzene $(\delta=7.20)$ as internal reference. $-{ }^{13} \mathrm{C}$ NMR spectra were recorded on a Bruker AW 250 instrument operating at $62.9 \mathrm{MHz}$. Chemical shifts are reported as $\delta$ values relative to the central signal due to $\mathrm{CDCl}_{3}(\delta=77.0)$ or $\mathrm{C}_{6} \mathrm{D}_{6}(\delta=128)$; the number of protons attached to each carbon were determined by the DEPT $(62.9 \mathrm{MHz})$ technique and are quoted as $(+)$ for $\mathrm{CH}_{3}$ and $\mathrm{CH}$ groups, (-) for $\mathrm{CH}_{2}$ groups and $\left(\mathrm{C}_{\text {quat }}\right)$ for quaternary carbon atoms. - IR spectra were recorded on a Bruker IFS 66 instrument. - Low-resolution EI mass spectra were recorded using a Finnigan MAT 95 instrument operating at an ionizing voltage of $70 \mathrm{eV}$ while high-resolution mass spectra were determined using the same instrument using preselected ion peak matching at $R \sim 10000$ to be within $\pm 2 \mathrm{ppm}$ of the exact masses. - Elemental analyses were performed by the Mikroanalytisches Labor des Instituts für Organische und Biomolekulare Chemie der Universität Göttingen, Germany. - Melting points are uncorrected. - Solvents for extraction and chromatography were of technical grade and distilled before use. - All reactions were carried out under dry nitrogen in oven- and/or flame-dried glassware. - Benzene, THF and diethyl ether were distilled from sodium. Dichloromethane was distilled from $\mathrm{CaH}_{2}$.

\section{Representative Procedures for the Preparation of Selected Compounds:}

6-(+)-(3S, 3aS, 7aS)-3-tert-Butoxy-3a-methyl-3a,4,5, 7a-tetrahydroindanyltributylstannane (trans2): $n$-Butyllithium $(15.1 \mathrm{~mL}, 36.5 \mathrm{mmol}, 2.42 \mathrm{M})$ was added dropwise at $-78^{\circ} \mathrm{C}$ to a solution of diisopropylamine $(3.69 \mathrm{~g}, 36.5 \mathrm{mmol})$ in THF $(150 \mathrm{~mL})$ and the resulting solution was stirred at $-78{ }^{\circ} \mathrm{C}$ for $0.5 \mathrm{~h}$. Tributyltin hydride $(8.98 \mathrm{~g}, 30.9 \mathrm{mmol})$ was then added slowly and the resulting mixture stirred for a further $0.5 \mathrm{~h}$ before being treated with copper(I) cyanide $(1.38 \mathrm{~g}, 15.4 \mathrm{mmol})$ at $-78^{\circ} \mathrm{C}$. The reaction mixture was slowly warmed to $-55^{\circ} \mathrm{C}$ until a yellow solution was formed. After the addition of a solution of $(+)-(3 S, 3 \mathrm{a} S, 7 \mathrm{a} R)$-6-trifluoromethanesulfonic acid 3-tertbutoxy-3a-methyl-7a,3a,4,5-tetrahydroindanyl ester $(5.00 \mathrm{~g}, 14.0 \mathrm{mmol})$ in THF (15 mL) the reaction mixture was warmed to $-25^{\circ} \mathrm{C}$, stirring continued at this temperature for $2 \mathrm{~h}$ then it was poured into saturated aqueous $\mathrm{NH}_{4} \mathrm{Cl}$ solution $(100 \mathrm{~mL})$ and extracted with pentane $(3 \times 90 \mathrm{~mL})$. The combined organic layers were washed with water $(2 \times 75 \mathrm{~mL})$, dried over $\mathrm{MgSO}_{4}$ then 
concentrated under reduced pressure. The residue so obtained was dissolved in ethyl acetate $(100 \mathrm{~mL})$ and the resulting solution treated with silver(I) acetate $(6.50 \mathrm{~g}, 39.0 \mathrm{mmol})$ whilst being maintained at $22^{\circ} \mathrm{C}$. After $2.5 \mathrm{~h}$, the reaction mixture was filtered through Celite and the filtrate washed with water $(2 \times 50 \mathrm{~mL})$ then dried over $\mathrm{MgSO}_{4}$. Concentration of the resulting solution under reduced pressure and subjection of the residue to column chromatography using neutral aluminum oxide ( $100 \mathrm{~g}$ with $5 \% \mathrm{v} / \mathrm{w}$ water, pentane, $4 \times 43 \mathrm{~cm}$ column) afforded the product trans$2\left(5.90 \mathrm{~g}, 85 \%, R_{\mathrm{f}}=0.5\right.$ in $20: 1 \mathrm{v} / \mathrm{v}$ pentane/diethyl ether), as a clear, colorless oil. - IR (film): $v$ $\max 2976,2956,2928,2872,2847,1606,1461,1419,1383,1359,1337,1290,1251,1195,1117$, 1070, 1021, 958, 897, 836, 689, $659 \mathrm{~cm}^{-1} .{ }^{1} \mathrm{H}$ NMR (250 MHz, $\left.\mathrm{CDCl}_{3}\right): \delta 0.73$ (s, $3 \mathrm{H}, \mathrm{CH}_{3}$ ), 0.82-1.01 (m, $14 \mathrm{H}, n$-Bu-CH $\left.3, n-\mathrm{Bu}-\mathrm{CH}_{2}\right), 1.14\left[\mathrm{~s}, 9 \mathrm{H}, \mathrm{C}\left(\mathrm{CH}_{3}\right)_{3}\right], 1.22-1.40(\mathrm{~m}, 7 \mathrm{H}, n$-Bu$\left.\mathrm{CH}_{2}\right), 1.42-1.58\left(\mathrm{~m}, 7 \mathrm{H}, n-\mathrm{Bu}-\mathrm{CH}_{2}\right), 1.65-1.83(\mathrm{~m}, 2 \mathrm{H}), 1.89-2.16(\mathrm{~m}, 2 \mathrm{H}), 2.20-2.41(\mathrm{~m}$, $4 \mathrm{H}), 3.43\left(\mathrm{dd},{ }^{3} J=6.8,{ }^{3} J=8.0 \mathrm{~Hz}, 1 \mathrm{H}, 3-\mathrm{H}\right), 5.72\left(\mathrm{~m}_{\mathrm{c}}, 1 \mathrm{H}, 7-\mathrm{H}\right) .-{ }^{13} \mathrm{C}$ NMR $(62.9 \mathrm{MHz}$, $\mathrm{CDCl}_{3}$, add. DEPT): $\delta 8.9\left(-, 3 \mathrm{C}, n-\mathrm{Bu}_{-} \mathrm{CH}_{2}\right), 11.0\left(+, \mathrm{CH}_{3}\right), 13.7\left(+, 3 \mathrm{C}, n-\mathrm{Bu}-\mathrm{CH}_{3}\right), 24.6(-$, $\left.\mathrm{CH}_{2}\right), 27.4\left(-, 3 \mathrm{C}, n-\mathrm{Bu}-\mathrm{CH}_{2}\right), 28.7$ [+, $\left.3 \mathrm{C}, \mathrm{C}\left(\mathrm{CH}_{3}\right)_{3}\right], 29.2\left(-, 3 \mathrm{C}, n-\mathrm{Bu}_{-} \mathrm{CH}_{2}\right), 30.8\left(-, \mathrm{CH}_{2}\right)$, $31.2\left(-, \mathrm{CH}_{2}\right), 35.6\left(-, \mathrm{CH}_{2}\right), 41.5\left(\mathrm{C}_{\text {quat }}, \mathrm{C}-3 \mathrm{a}\right), 44.8(+, \mathrm{CH}, \mathrm{C}-7 \mathrm{a}), 72.1\left[\mathrm{C}_{\text {quat }}, C\left(\mathrm{CH}_{3}\right)_{3}\right], 79.6$ (+, CH, C-3), 138.3 (C quat, $_{1}$ C-6), 141.7 (+, CH, C-7). - MS (70 eV), m/z (\%): 443/442/441/440/ 439/438/437 (16/24/100/44/83/33/46) [M-C $\left.\mathrm{C}_{4} \mathrm{H}_{9} \cdot\right]^{+}, 293 / 292 / 291 / 290 / 289 / 288 / 287$ (1/1/6/3/5/2/3), 239/237/236/235/234/233/232/231 (15/12/11/99/32/76/26/42), 180/179/178/177/176/175 (11/88/ 83/53/26/12), 135 (5), 122/121/120/119/118/117 (1/5/26/20/12/10), 91 (3), 57 (84), 41 (22). - Anal. Calcd for $\mathrm{C}_{26} \mathrm{H}_{50} \mathrm{OSn}$ (497.37): C 62.78, H 10.13; found C 62.86, H 10.01 .

Trifluoromethanesulfonic acid 1,4-dioxaspiro[4,5]dec-7-en-8-yl ester (3): Sodium bis(trimethylsilyl) amide (13.4 mL of a $2.00 \mathrm{M}$ solution in THF, $26.8 \mathrm{mmol}$ ) was added to a magnetically stirred solution of 1,4-dioxaspiro[4,5]decan-8-one $(4.00 \mathrm{~g}, 25.6 \mathrm{mmol})$ in diethyl ether $(300 \mathrm{~mL})$ maintained at $-20^{\circ} \mathrm{C}$. After $1 \mathrm{~h}$ the reaction mixture was treated with trifluoromethanesulfonic acid anhydride $(4.52 \mathrm{~mL}, 26.9 \mathrm{mmol})$ and after a further $16 \mathrm{~h}$, during which time the temperature was raised to $22{ }^{\circ} \mathrm{C}$, the reaction mixture was washed with saturated aqueous sodium bicarbonate solution $(1 \times 50 \mathrm{~mL})$ and water $(1 \times 50 \mathrm{~mL})$. The combined aqueous phases were reextracted with diethyl ether $(2 \times 50 \mathrm{~mL})$ and the combined organic phases then dried over $\mathrm{MgSO}_{4}$ and concentrated under reduced pressure. The ensuing residue was subjected to column chromatography on silica gel $(25 \mathrm{~g}, 5: 1 \mathrm{v} / \mathrm{v}$ pentane/diethyl ether, $3 \times 35 \mathrm{~cm}$ column $)$ 
to yield the title compound $3\left(7.08 \mathrm{~g}, 96 \%, R_{\mathrm{f}}=0.4\right)$ as a clear, colorless oil. The spectral data recorded on this material were consistent with those reported in the literature. ${ }^{(1)}$

(1'S, 3a' $\left.\mathrm{S}, 7 a^{\prime} \mathrm{S}\right)-8-\left(1^{\prime}\right.$-tert-Butoxy-7a'-methyl-2',3',3a', 6', 7', 7a'-hexahydro-1'H-inden-5'-yl)-1,4dioxaspiro[4.5]dec-7-ene (trans-4): A Pyrex bottle containing a magnetic stirring bar was charged with a solution of cyclohexene $3(288 \mathrm{mg}, 1.00 \mathrm{mmol})$ and a solution of bicycloalkenylstannane trans-2 (597 mg, $1.20 \mathrm{mmol}$ ) in 1-methyl-2-pyrrolidinone (NMP, $5 \mathrm{~mL}$ ). After purging the resulting solution with argon in an ultrasonic bath for $5 \mathrm{~min}, \mathrm{Pd}_{2}\left(\mathrm{dba}_{3} \cdot \mathrm{CHCl}_{3}\right.$ (54.0 mg, $52.2 \mu \mathrm{mol}), \mathrm{LiCl}(127 \mathrm{mg}, 3.00 \mathrm{mmol})$ and $\mathrm{CuI}(30.0 \mathrm{mg}, 158 \mu \mathrm{mol})$ were added. Before carefully sealing the bottle with a screw cap, the resulting solution was again placed in an ultrasonic bath and purged with argon for $5 \mathrm{~min}$. After stirring the reaction mixture vigorously for $12 \mathrm{~h}$ at $90{ }^{\circ} \mathrm{C}$ it was cooled, poured into diethyl ether $(50 \mathrm{~mL})$ and then washed with water $(2 \times 25 \mathrm{~mL})$. The combined aqueous phases were reextracted with diethyl ether $(2 \times 30 \mathrm{~mL})$, the combined organic extracts treated with saturated aqueous KF solution $(1 \times 30 \mathrm{~mL})$ and then dried with $\mathrm{MgSO}_{4}$, filtered and concentrated under reduced pressure. The ensuing light-yellow oil was subjected to column chromatography on silica gel $(27 \mathrm{~g}, 10: 1 \mathrm{v} / \mathrm{v}$ pentane/diethyl ether, $3.0 \times 35 \mathrm{~cm}$ column) and thus yielding the tricyclic butadiene trans $-4\left(265 \mathrm{mg}, 77 \%, R_{\mathrm{f}}=0.4\right)$ as a colorless wax. - IR (film): $v_{\max }$ 2975, 2930, 2873, 1465, 1457, 1388, 1377, 1362, 1341, 1252, $1197,1165,1118,1059,1015,982,946,907,859,734,701 \mathrm{~cm}^{-1} .-{ }^{1} \mathrm{H}$ NMR $\left(250 \mathrm{MHz}, \mathrm{CDCl}_{3}\right)$ : ઈ $0.69\left(\mathrm{~s}, 3 \mathrm{H}, \mathrm{CH}_{3}\right), 1.17$ [s, $\left.9 \mathrm{H}, \mathrm{C}\left(\mathrm{CH}_{3}\right)_{3}\right], 1.22-1.62(\mathrm{~m}, 3 \mathrm{H}), 1.75-2.21(\mathrm{~m}, 6 \mathrm{H}), 2.24-2.33$ (m, $6 \mathrm{H}), 3.49$ (dd, $\left.{ }^{3} J=11.4,{ }^{3} J=8.0 \mathrm{~Hz}, 1 \mathrm{H}, 1-\mathrm{H}\right), 3.98$ (s, $\left.4 \mathrm{H}, 2^{\prime}-\mathrm{H}, 3^{\prime}-\mathrm{H}\right), 5.22-5.76$ (m, $2 \mathrm{H}$, 4'-H, 7-H). $-{ }^{13} \mathrm{C}$ NMR (75.5 MHz, $\mathrm{CDCl}_{3}$, add. APT): $\delta 11.0\left(+, \mathrm{CH}_{3}\right), 24.5\left(-, \mathrm{CH}_{2}\right), 24.8(-$, $\left.2 \mathrm{C}, \mathrm{CH}_{2}\right), 28.7$ [+, $\left.3 \mathrm{C}, \mathrm{C}\left(\mathrm{CH}_{3}\right)_{3}\right], 31.2\left(-, \mathrm{CH}_{2}\right), 31.7\left(-, \mathrm{CH}_{2}\right), 34.2\left(-, \mathrm{CH}_{2}\right), 36.0\left(-, \mathrm{CH}_{2}\right)$, 42.0 (-, $\left.\mathrm{C}_{\text {quat, }} \mathrm{C}-7^{\prime}\right), 44.4$ (+, CH, C-3'), $64.4\left(-, \mathrm{CH}_{2}, \mathrm{OCH}_{2} \mathrm{CH}_{2} \mathrm{O}\right), 64.4\left(-, \mathrm{CH}_{2}, \mathrm{OCH}_{2} \mathrm{CH}_{2} \mathrm{O}\right)$, $72.2\left[-, \mathrm{C}_{\text {quat }}, \mathrm{C}\left(\mathrm{CH}_{3}\right)_{3}\right], 79.4\left(+, \mathrm{CH}, \mathrm{C}-1^{\prime}\right), 108.0\left(-, \mathrm{C}_{\text {quat }}, \mathrm{C}-5\right), 118.5\left(-, \mathrm{C}_{\text {quat }}\right), 123.8$ (-, $\left.\mathrm{C}_{\text {quat }}\right)$,

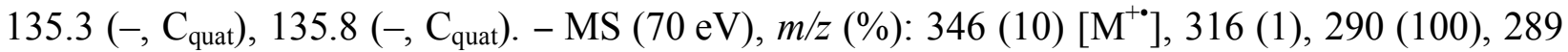
(55), 272 (5), 228 (26), 203 (22), 185 (10), 171 (6), 159 (9), 131 (12), 105 (11), 91 (10), 86 (22), 79 (4), 57 (38), 41 (9). $-\mathrm{C}_{22} \mathrm{H}_{34} \mathrm{O}_{3}$ (346.5). - ESI-HRMS: calcd for $\left[\mathrm{M}+\mathrm{H}^{+}\right] 347.2581$ (correct HRMS). 
(6S, 7S, 5R, 8S, 13R, 14S, 17S)-17-tert-Butoxy-13-methyl-1',4'-spiro[4',3]dioxolan-2, 3, 4, 5, 6, 7,8, 11,12,13,14,15,16,17-tetradecahydro-1H-cyclopenta[a]phenanthrene-6,7-dicarbonitrile (trans5): A Pyrex bottle containing a magnetic stirring bar was charged with a solution of tricyclic diene trans-4 (350 mg, $1.01 \mathrm{mmol})$ and fumarodinitrile $(117 \mathrm{mg}, 1.50 \mathrm{mmol})$ in toluene $(2.00 \mathrm{~mL})$. The reaction mixture was purged with argon for $5 \mathrm{~min}$, sealed and then heated at $110^{\circ} \mathrm{C}$ for $16 \mathrm{~h}$. The cooled reaction mixture was concetrated under reduced pressure and the residue subjected to column chromatography on silica gel (25 g, 1:1 v/v pentane/diethyl ether, $1.0 \times 20 \mathrm{~cm}$ column). Concentration of the relevant fractions provided the title compound trans $\mathbf{- 5}$ $\left(357 \mathrm{mg}, 84 \% R_{\mathrm{f}}=0.4\right)$ as colorless crystals, $\mathrm{mp} 183-184^{\circ} \mathrm{C}$. Crystals suitable for X-ray diffraction analysis were grown from 1:1 v/v pentane/diethyl ether by slow evaporation of the solvent. $-[\alpha]_{\mathrm{D}}{ }^{20}=+36.5,(c=1.06, \mathrm{MeOAc})$. - IR (film): $v_{\max } 2973,2931,2882,2296,1700$, 1653, 1635, 1472, 1457, 1394, 1362, 1246, 1196, 1143, 1107, 1094, 1068, 1041, 1017, 982, 946, 906, $881 \mathrm{~cm}^{-1} .-{ }^{1} \mathrm{H}$ NMR $\left(250 \mathrm{MHz}, \mathrm{CDCl}_{3}\right): \delta 0.86\left(\mathrm{~s}, 3 \mathrm{H}, \mathrm{CH}_{3}\right), 1.16\left[\mathrm{~s}, 9 \mathrm{H}, \mathrm{C}\left(\mathrm{CH}_{3}\right)_{3}\right]$, 1.32-2.13 (m, $13 \mathrm{H}), 2.29\left(\mathrm{~m}_{\mathrm{c}}, 1 \mathrm{H}\right), 2.55\left(\mathrm{~m}_{\mathrm{c}}, 1 \mathrm{H}\right), 2.71-2.86(\mathrm{~m}, 2 \mathrm{H}), 3.00-3.14(\mathrm{~m}, 2 \mathrm{H})$, $3.46\left(\mathrm{t},{ }^{3} J=8.3 \mathrm{~Hz}, 1 \mathrm{H}, 17-\mathrm{H}\right), 3.98\left(\mathrm{~m}_{\mathrm{c}}, 4 \mathrm{H}, \mathrm{OCH}_{2} \mathrm{CH}_{2} \mathrm{O}\right) .-{ }^{13} \mathrm{C} \mathrm{NMR}\left(75.6 \mathrm{MHz}, \mathrm{CDCl}_{3}\right.$, add. APT): $\left.\delta 10.8\left(+, \mathrm{CH}_{3}\right), 23.6\left(-, \mathrm{CH}_{2}\right), 24.1\left(-, \mathrm{CH}_{2}\right), 26.8\left(-, \mathrm{CH}_{2}\right), 28.7\left[+, 3 \mathrm{C}, \mathrm{C}_{(} \mathrm{CH}_{3}\right)_{3}\right]$, $29.5(+, \mathrm{CH}), 30.9\left(-, \mathrm{CH}_{2}\right), 31.9\left(+, \mathrm{CH}_{2}\right), 35.4\left(-, \mathrm{CH}_{2}\right), 36.1(+, \mathrm{CH}), 36.1\left(-, \mathrm{CH}_{2}\right), 37.6(+$, $\mathrm{CH}), 42.5\left(-, \mathrm{CH}_{2}\right), 42.6\left(-, \mathrm{CH}_{2}\right), 47.1$ (+, $\left.\mathrm{CH}\right), 64.5\left(-, \mathrm{CH}_{2}, \mathrm{OCH}_{2} \mathrm{CH}_{2} \mathrm{O}\right), 64.6\left(-, \mathrm{CH}_{2}\right.$, $\left.\mathrm{OCH}_{2} \mathrm{CH}_{2} \mathrm{O}\right), 72.5\left[-, \mathrm{C}_{\text {quat }}, \mathrm{C}\left(\mathrm{CH}_{3}\right)_{3}\right], 80.0(+, \mathrm{CH}, \mathrm{C}-17), 108.1\left(-, \mathrm{C}_{\text {quat }}, \mathrm{OCO}\right), 118.3\left(-, \mathrm{C}_{\text {quat }}\right.$, $\mathrm{CN}), 119.4$ (-, $\left.\mathrm{C}_{\text {quat }}, \mathrm{CN}\right), 127.5$ (-, $\left.\mathrm{C}_{\text {quat }}\right), 128.1$ (-, $\left.\mathrm{C}_{\text {quat }}\right) .-\mathrm{MS}(70 \mathrm{eV}), \mathrm{m} / \mathrm{z}$ (\%): 424 (29) $\left[\mathrm{M}^{+\bullet}\right.$ ], 396 (6), 368 (51), 350 (31), 323 (26), 305 (14), 279 (42), 262 (14), 235 (6), 194 (2), 180 (8), 168 (4), 129 (4), 99 (10), 86 (56), 57 (100), 41 (14). - $\mathrm{C}_{26} \mathrm{H}_{36} \mathrm{O}_{3} \mathrm{~N}_{2}$ (424.6). - ESI-HRMS: calcd for $\left[\mathrm{M}+\mathrm{H}^{+}\right] 425.2804$ (correct mass).

(3aR,3bS,9aS, 10S, 12aS, 12bR, 12cS)-10-tert-Butoxy-2,9a-dimethyl-3b, 6, 7,8,9,9a,10,11,12,12a, 12b,12c-dodecahydro-3aH,4H-2-azadicyclopenta[a,l]phenanthrene-1,3,5-trione (monodeprotected derivative of trans-6): $p$-Toluenesulfonic acid (76.1 $\mathrm{mg}, 0.400 \mathrm{mmol})$ was added to a solution of the steroid analogue trans-6 $(540 \mathrm{mg}, 1.18 \mathrm{mmol})$ in acetone $(25 \mathrm{~mL})$ and water $(100 \mu \mathrm{L})$. The resulting solution was stirred magnetically at $23{ }^{\circ} \mathrm{C}$ for $24 \mathrm{~h}$ then concentrated under reduced pressure and the residue was dissolved in diethyl ether $(75 \mathrm{~mL})$. The resulting solution was washed with a saturated aqueous $\mathrm{NaHCO}_{3}$ solution $(1 \times 20 \mathrm{~mL})$ then dried over 
$\mathrm{MgSO}_{4}$, filtered and concentrated under reduced pressure. The residue so-obtained was subjected to column chromatography on silica gel $(30 \mathrm{~g}, 1: 2 \mathrm{v} / \mathrm{v}$ pentane/diethyl ether, $3.0 \times 30 \mathrm{~cm}$ column) to yield the mono-deprotected derivative of trans-6 $\left(483 \mathrm{mg}, 99 \%, R_{\mathrm{f}}=0.35\right)$ as a colorless wax. - IR (film): $v_{\max } 2967,2930,2871,1768,1695,1653,1457,1436,1385,1362,1336,1288,1268$, 1226, 1198, 1131, 1095, 1081, 1064, 980, 963, 900, $839 \mathrm{~cm}^{-1} .-{ }^{1} \mathrm{H} \mathrm{NMR}\left(250 \mathrm{MHz}, \mathrm{CDCl}_{3}\right): \delta$ $0.69\left(\mathrm{~s}, 3 \mathrm{H}, \mathrm{CH}_{3}\right), 1.16$ [s, $\left.9 \mathrm{H}, \mathrm{C}\left(\mathrm{CH}_{3}\right)_{3}\right], 1.21-1.73(\mathrm{~m}, 8 \mathrm{H}), 1.93-2.13(\mathrm{~m}, 1 \mathrm{H}), 2.19-2.52$ (m, 5 H), 2.56-2.73 (m, 2 H), 2.96-3.09 (m, $2 \mathrm{H}), 2.90$ (s, $\left.3 \mathrm{H}, \mathrm{N}-\mathrm{CH}_{3}\right), 3.49\left(\mathrm{~m}_{\mathrm{C}}, 1 \mathrm{H}\right), 3.58$ (t, $\left.{ }^{3} J=8.7 \mathrm{~Hz}, 1 \mathrm{H}, 10-\mathrm{H}\right) .-{ }^{13} \mathrm{C}$ NMR $\left(75.5 \mathrm{MHz}, \mathrm{C}_{6} \mathrm{D}_{6}\right.$, add. APT): $\delta 11.6\left(+, \mathrm{CH}_{3}\right), 22.9(-$, $\left.\mathrm{CH}_{2}\right), 23.2\left(-, \mathrm{CH}_{2}\right), 24.3(+, \mathrm{CH}), 25.3\left(-, \mathrm{CH}_{2}\right), 28.5\left[+, 3 \mathrm{C}, \mathrm{C}\left(\mathrm{CH}_{3}\right)_{3}\right], 31.8\left(-, \mathrm{CH}_{2}\right), 34.0(-$, $\left.\mathrm{CH}_{2}\right), 34.3(+, \mathrm{CH}), 38.2\left(-, \mathrm{CH}_{2}\right), 40.7(+, \mathrm{CH}), 40.8(+, \mathrm{CH}), 41.4\left(-, \mathrm{CH}_{2}\right), 42.0(+, \mathrm{CH}), 42.8$ $\left(\mathrm{C}_{\text {quat }}, \mathrm{C}-9 \mathrm{a}\right), 43.8(+, \mathrm{CH}), 72.2\left[\mathrm{C}_{\text {quat }}, C\left(\mathrm{CH}_{3}\right)_{3}\right], 80.8(+, \mathrm{CH}, \mathrm{C}-10), 130.6\left(-, \mathrm{C}_{\text {quat }}\right), 131.0(-$, $\left.\mathrm{C}_{\text {quat }}\right), 177.1$ (-, $\left.\mathrm{C}_{\text {quata }}, \mathrm{NC}=\mathrm{O}\right), 177.5\left(-, \mathrm{C}_{\text {quat, }}, \mathrm{NC}=\mathrm{O}\right), 209.3\left(-, \mathrm{C}_{\text {quat }}, \mathrm{C}=\mathrm{O}\right) .-\mathrm{MS}(70 \mathrm{eV}), \mathrm{m} / \mathrm{z}$ (\%): 413 (5) [ $\left.\mathrm{M}^{+*}\right], 371$ (6), 357 (100), 356 (21), 339 (82), 312 (20), 300 (17), 295 (11), 246 (28), 227 (20), 201 (6), 166 (12), 122 (4), 113 (13), 61 (8), 57 (38), 43 (29). - $\mathrm{C}_{25} \mathrm{H}_{35} \mathrm{NO}_{4}$ (413.6): - ESI-HRMS: calcd for $\left[\mathrm{M}+\mathrm{H}^{+}\right] 414.2639$ (correct HRMS).

(3aR,3bS,9aS, 10S, 12aS, 12bR,12cS)-10-Hydroxy-2,9a-dimethyl-3b, 6, 7,8,9,9a, 10,11,12,12a, 12b,12c-dodecahydro-3aH,4H-2-azadicyclopenta[a, l]phenanthrene-1,3,5-trione (trans-12):

Trifluoroacetic acid $(3.00 \mathrm{~mL})$ was added to a solution of the mono-deprotected derivative of trans-6 $(237 \mathrm{mg}, 0.573 \mathrm{mmol})$ in methylene chloride $(27.0 \mathrm{~mL})$ and the ensuing mixture stirred at $23{ }^{\circ} \mathrm{C}$ for $2 \mathrm{~h}$ then concentrated under reduced pressure and the residue dissolved in diethyl ether $(30 \mathrm{~mL})$. The resulting solution was washed with saturated aqueous sodium bicarbonate solution $(1 \times 10 \mathrm{~mL})$ then dried over $\mathrm{MgSO}_{4}$, filtered and concentrated under reduced pressure. The residue so-obtained was purified by column chromatography on silica gel ( $25 \mathrm{~g}, 97: 3 \mathrm{v} / \mathrm{v}$ diethyl ether/methanol, $1.0 \times 20 \mathrm{~cm}$ column) to yield the product trans $-12\left(186 \mathrm{mg}, 91 \%, R_{\mathrm{f}}=0.3\right)$ as a colorless solid mp $169-173{ }^{\circ} \mathrm{C} .-[\alpha]_{\mathrm{D}}{ }^{20}=-16.1^{\circ}(c=0.98, \mathrm{MeOAc}) .-$ IR (film): $v_{\max } 3370$, 2955, 2876, 1768, 1684, 1653, 1559, 1437, 1382, 1371, 1286, 1265, 1138, 1046, 1034, 983, 911 , 881, $844 \mathrm{~cm}^{-1} .-{ }^{1} \mathrm{H}$ NMR $\left(300 \mathrm{MHz}, \mathrm{CDCl}_{3}\right): \delta 0.67\left(\mathrm{~s}, 3 \mathrm{H}, \mathrm{CH}_{3}\right), 1.18-1.78(\mathrm{~m}, 6 \mathrm{H})$, 2.01-2.27 (m, $4 \mathrm{H}), 2.28-2.51$ (m, $3 \mathrm{H}), 2.54-2.70$ (m, $4 \mathrm{H}), 2.85$ (s, $3 \mathrm{H}, \mathrm{NCH}_{3}$ ), 2.90-3.05 (m, $2 \mathrm{H}), 3.46\left(\mathrm{dd},{ }^{3} J=20,{ }^{3} J=12 \mathrm{~Hz}, 1 \mathrm{H}\right), 3.82\left(\mathrm{t},{ }^{3} J=8.0 \mathrm{~Hz}, 1 \mathrm{H}, 10-\mathrm{H}\right) .-{ }^{13} \mathrm{C}$ NMR $(75.6 \mathrm{MHz}$, $\mathrm{CDCl}_{3}$ additional H,H-COSY, APT, HSQC, HMBC, NOESY): $\delta 11.4\left(+, \mathrm{CH}_{3}\right), 22.9\left(-, \mathrm{CH}_{2}\right)$, 
$23.4\left(-, \mathrm{CH}_{2}\right), 24.3\left(-, \mathrm{CH}_{2}\right), 24.7\left(+, \mathrm{CH}_{3}, \mathrm{NCH}_{3}\right), 30.6\left(-, \mathrm{CH}_{2}\right), 33.1\left(-, \mathrm{CH}_{2}\right), 34.4(+, \mathrm{CH})$, $38.1\left(-, \mathrm{CH}_{2}\right), 40.6(+, \mathrm{CH}), 40.7\left(-, \mathrm{CH}_{2}\right), 41.2\left(-, \mathrm{C}_{\text {quat }}, \mathrm{C}-9 \mathrm{a}\right), 41.9(+, \mathrm{CH}), 42.7(+, \mathrm{CH}), 43.7$ $(+, \mathrm{CH}), 81.5$ (+, $\mathrm{CH}, \mathrm{C}-10), 130.0$ (-, $\left.\mathrm{C}_{\text {quat }}\right), 131.3$ (-, $\left.\mathrm{C}_{\text {quat }}\right), 177.3$ (-, $\left.\mathrm{C}_{\text {quat }}\right), 177.8$ (-, $\left.\mathrm{C}_{\text {quat }}\right)$,

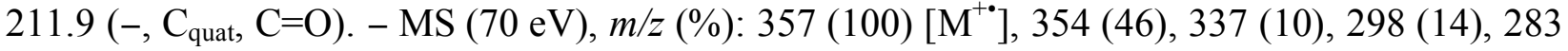
(10), 246 (100), 221 (82), 185 (11), 167 (15), 143 (14), 129 (20), 113 (62), 79 (18), 55 (30). - $\mathrm{C}_{21} \mathrm{H}_{27} \mathrm{NO}_{4}$ (357.4). - ESI-HRMS: calcd for $\left[\mathrm{M}+\mathrm{H}^{+}\right] 358.2012$ (correct HRMS).

(13R, 14S, 17S)-17-tert-Butoxy-13-methyl-1',4'-spiro[4',3]dioxolan-2,3,4,5, 8, 11,12,13,14,15,16, 17-dodecahydro-1H-cyclopenta[a]phenanthrene-6,7-dicarboxylic acid dimethyl ester (trans-14): A solution of the aromatic steroid analogue trans-7 (50.0 mg, $0.102 \mathrm{mmol})$ and DDQ (24.3 mg, $0.107 \mathrm{mmol})$ in 1,4 -dioxane $(1.50 \mathrm{~mL})$ was stirred at $100{ }^{\circ} \mathrm{C}$ for $1 \mathrm{~h}$. The cooled reaction mixture was concentrated under reduced pressure and the residue so-obtained subjected to column chromatography on silica gel $(20 \mathrm{~g}, 1: 1 \mathrm{v} / \mathrm{v}$ pentane/diethyl ether, $1.0 \times 20 \mathrm{~cm}$ column $)$ to yield the product trans-14 $\left(34.2 \mathrm{mg}, 69 \%, R_{\mathrm{f}}=0.4\right)$ as a colorless wax. - IR (film): $v_{\max } 3064,2970$, $2928,1702,1650,1472,1445,1410,1373,1302,1244,1180,1106,1066,944,915,854 \mathrm{~cm}^{-1}$.${ }^{1} \mathrm{H}$ NMR $\left(300 \mathrm{MHz}, \mathrm{CDCl}_{3}\right): \delta 0.60\left(\mathrm{~s}, 3 \mathrm{H}, \mathrm{CH}_{3}\right), 1.16\left[\mathrm{~s}, 9 \mathrm{H}, \mathrm{C}\left(\mathrm{CH}_{3}\right)_{3}\right], 1.22-1.71(\mathrm{~m}, 3 \mathrm{H})$, 1.88-2.05 (m, 4 H), 2.59-2.88 (m, $7 \mathrm{H}), 3.21\left(\mathrm{~d},{ }^{3} J=15.9 \mathrm{~Hz}, 1 \mathrm{H}\right), 3.55\left(\mathrm{t},{ }^{3} J=6.9 \mathrm{~Hz}, 1 \mathrm{H}, 17-\right.$ $\mathrm{H}), 3.78\left(\mathrm{~s}, 3 \mathrm{H}, \mathrm{OCH}_{3}\right), 3.83\left(\mathrm{~s}, 3 \mathrm{H}, \mathrm{OCH}_{3}\right), 3.98\left(\mathrm{~m}_{\mathrm{C}}, 4 \mathrm{H}, \mathrm{OCH}_{2} \mathrm{CH}_{2} \mathrm{O}\right) .-{ }^{13} \mathrm{C} \mathrm{NMR}$ (75.6 MHz, $\mathrm{CDCl}_{3}$, add. APT): $\delta 11.4\left(+, \mathrm{CH}_{3}\right), 23.6\left(-, \mathrm{CH}_{2}\right), 25.6\left(-, \mathrm{CH}_{2}\right), 26.2\left(-, \mathrm{CH}_{2}\right), 28.7$ [+, $\left.3 \mathrm{C}, \mathrm{C}\left(\mathrm{CH}_{3}\right)_{3}\right], 31.2\left(-, \mathrm{CH}_{2}\right), 31.4\left(-, \mathrm{CH}_{2}\right), 33.4\left(-, \mathrm{CH}_{2}\right), 37.3\left(-, \mathrm{CH}_{2}\right), 42.3(+, \mathrm{CH}), 45.4$ $\left(-, \mathrm{C}_{\text {quat }}, \mathrm{C}-13\right), 52.5\left(+, 2 \mathrm{C}, \mathrm{OCH}_{3}\right), 64.5\left(-, 2 \mathrm{C}, \mathrm{OCH}_{2} \mathrm{CH}_{2} \mathrm{O}\right), 72.5\left[\mathrm{C}_{\text {quat }}, \mathrm{C}\left(\mathrm{CH}_{3}\right)_{3}\right], 79.3(+$, $\mathrm{CH}, \mathrm{C}-17), 107.4\left(-, \mathrm{C}_{\text {quat }}, \mathrm{C}-3\right), 129.2$ (-, $\left.\mathrm{C}_{\text {quat }}\right), 129.8$ (-, $\left.\mathrm{C}_{\text {quat }}\right), 130.0$ (-, $\left.\mathrm{C}_{\text {quat }}\right), 135.0$ (-, $\left.\mathrm{C}_{\text {quat }}\right)$, 135.8 (-, $\left.\mathrm{C}_{\text {quat }}\right), 138.7$ (-, $\left.\mathrm{C}_{\text {quat }}\right), 169.0$ (-, $\left.\mathrm{C}_{\text {quat }}, \mathrm{C}=\mathrm{O}\right), 170.5$ (-, $\left.\mathrm{C}_{\text {quat }}, \mathrm{C}=\mathrm{O}\right) .-\mathrm{ESI}-\mathrm{MS}(\mathrm{MeOH}$, $\mathrm{NH}_{3}$ ), $m / z$ (\%): 990 (49), 504 (100) $\left[\mathrm{M}+\mathrm{NH}_{4}^{+}\right], 455$ (75), 397 (11). $-\mathrm{C}_{28} \mathrm{H}_{38} \mathrm{O}_{7}$ (486.6). - ESIHRMS: calcd for $\left[\mathrm{M}+\mathrm{NH}_{4}^{+}\right] 504.2960$ (correct HRMS). 


\section{Spectral Data for Remaining Compounds:}

cis-2: IR (film): $v_{\max } 2957,2925,2871,1609,1464,1418,1387,1376,1361,1292,1198,1072$, 1020, 902, 874, $689 \mathrm{~cm}^{-1}$. - ${ }^{1} \mathrm{H}$ NMR $\left(250 \mathrm{MHz}, \mathrm{C}_{6} \mathrm{D}_{6}\right)$ : $\delta$ 0.82-1.04 (m, $15 \mathrm{H}, n$-Bu-CH $3, n$-Bu$\left.\mathrm{CH}_{2}\right), 1.13\left(\mathrm{~s}, 3 \mathrm{H}, \mathrm{CH}_{2}\right), 1.15$ [s, $\left.9 \mathrm{H}, \mathrm{C}\left(\mathrm{CH}_{3}\right)_{3}\right], 1.24-1.79(\mathrm{~m}, 16 \mathrm{H}), 1.89-2.16(\mathrm{~m}, 2 \mathrm{H})$, 2.18-2.27 (m, $1 \mathrm{H}), 2.33\left(\mathrm{~m}_{\mathrm{c}}, 2 \mathrm{H}\right), 3.66\left(\mathrm{t},{ }^{3} J=6.8 \mathrm{~Hz}, 1 \mathrm{H}, 3-\mathrm{H}\right), 5.85-5.95(\mathrm{~m}, 1 \mathrm{H}, 7-\mathrm{H})$. $-{ }^{13} \mathrm{C}$ NMR (62.9 MHz, $\mathrm{C}_{6} \mathrm{D}_{6}$, add. DEPT): $\delta 9.3\left(-, n-\mathrm{Bu}_{-} \mathrm{CH}_{2}\right), 14.0\left(+, n-\mathrm{Bu}-\mathrm{CH}_{3}\right), 21.8(+$, $\left.\mathrm{CH}_{3}\right), 27.8\left[+, 3 \mathrm{C}, \mathrm{C}\left(\mathrm{CH}_{3}\right)_{3}\right], 28.9\left(-, n-\mathrm{Bu}_{-} \mathrm{CH}_{2}\right), 29.1\left(-, \mathrm{CH}_{2}\right), 29.3\left(-, \mathrm{CH}_{2}\right), 29.7(-, n-$ Bu- $\left.\mathrm{CH}_{2}\right), 31.1\left(-, \mathrm{CH}_{2}\right), 32.8\left(-, \mathrm{CH}_{2}\right), 42.2\left(\mathrm{C}_{\text {quat, }}, \mathrm{C}-3 \mathrm{a}\right), 46.2(+, \mathrm{C}-7 \mathrm{a}), 72.4\left[\mathrm{C}_{\text {quat }}, \mathrm{C}\left(\mathrm{CH}_{3}\right)_{3}\right]$, $76.7 \quad(+, \quad \mathrm{C}-3), \quad 137.4 \quad\left(\mathrm{C}_{\text {quat }}, \quad \mathrm{C}-6\right), \quad 142.7 \quad(+, \quad \mathrm{C}-7) . \quad-\mathrm{MS}(70 \mathrm{eV}), \quad m / z \quad(\%)$ : 443/442/441/440/439/438/437 (16/24/100/44/83/33/46), 387/386/385/384/383/382/381 (1/2/13/5/ 16/5/6), 331/330/329/327/326/325/324 (2/1/12/4/11/4/5), 293/292/291/290/289/288/287 (1/1/6/3/ 5/2/3), 237/236/235/234/233/232/231 (1/1/4/2/3/2/1), 179/178/177/176/175 (2/1/4/1/2), 136 (2), 122/121/120/119/118/117 (1/5/2/4/2/3), 91 (3), 57 (21), 41 (3). - Anal. Calcd for $\mathrm{C}_{26} \mathrm{H}_{50} \mathrm{OSn}$ (497.4): C 62.78, H 10.13; found C 62.83, H 10.17.

cis-4: IR (film): $v_{\max } 2971,2929,2873,1621,1493,1463,1447,1433,1422,1387,1362,1303$, $1255,1224,1198,1140,1117,1061,1058,1040,1017,994,950,897,875,861 \mathrm{~cm}^{-1}$. - ${ }^{1} \mathrm{H}$ NMR $\left(250 \mathrm{MHz}, \mathrm{C}_{6} \mathrm{D}_{6}\right): \delta 1.15$ [s, $\left.9 \mathrm{H}, \mathrm{C}\left(\mathrm{CH}_{3}\right)_{3}\right], 1.17\left(\mathrm{~s}, 3 \mathrm{H}, \mathrm{CH}_{3}\right), 1.21-1.48(\mathrm{~m}, 2 \mathrm{H}), 1.58-1.78$ (m, $3 \mathrm{H}), 1.87\left(\mathrm{~m}_{\mathrm{c}}, 2 \mathrm{H}\right), 1.93-2.20(\mathrm{~m}, 3 \mathrm{H}), 2.23\left(\mathrm{~m}_{\mathrm{c}}, 4 \mathrm{H}\right), 2.24-2.33(\mathrm{~m}, 1 \mathrm{H}), 3.53-3.62(\mathrm{~m}$, $\left.5 \mathrm{H}, 1-\mathrm{H}, 2^{\prime}-\mathrm{H}, 3^{\prime}-\mathrm{H}\right), 5.69\left(\mathrm{~m}_{\mathrm{c}}, 1 \mathrm{H}, 7^{\prime}-\mathrm{H}\right), 5.39\left(\mathrm{~m}_{\mathrm{c}}, 1 \mathrm{H}, 4-\mathrm{H}\right) .-{ }^{13} \mathrm{C} \mathrm{NMR}\left(75.5 \mathrm{MHz}, \mathrm{CDCl}_{3}\right.$ add. APT): $\delta 21.5\left(+, \mathrm{CH}_{3}\right), 22.8\left(-, \mathrm{CH}_{2}\right), 25.4\left(-, \mathrm{CH}_{2}\right), 28.8$ [+, $\left.3 \mathrm{C}, \mathrm{C}\left(\mathrm{CH}_{3}\right)_{3}\right], 29.4\left(-, \mathrm{CH}_{2}\right)$, $30.9\left(-, \mathrm{CH}_{2}\right), 31.8\left(-, \mathrm{CH}_{2}\right), 33.1\left(-, \mathrm{CH}_{2}\right), 36.6\left(-, \mathrm{CH}_{2}\right), 42.2\left(-, \mathrm{C}_{\text {quat, }}, \mathrm{C}-7 \mathrm{a}\right), 44.9(+, \mathrm{CH}$, C-3a), 64.3 (-, $\mathrm{CH}_{2}, 2 \mathrm{C}, \mathrm{C}-2$ ', C-3'), 72.4 [-, $\left.\mathrm{C}_{\text {quat }}, C\left(\mathrm{CH}_{3}\right)_{3}\right], 76.9$ (+, $\left.\mathrm{CH}, \mathrm{C}-1\right), 108.2\left(-, \mathrm{C}_{\text {quat, }}\right.$ C-5'), 119.2 (+, CH), 126.8 (+, CH), 133.9 (-, $\left.\mathrm{C}_{\text {quat }}\right), 136.4\left(-, \mathrm{C}_{\text {quat }}\right) .-\mathrm{MS}(70 \mathrm{eV}), \mathrm{m} / z(\%): 346$ (3) $\left[\mathrm{M}^{+*}\right], 321$ (1), 306 (3), 290 (100), 289 (46), 245 (4), 228 (30), 203 (9), 185 (9), 159 (11), 145 (13), 131 (20), 97 (19), 91 (22), 86 (42), 79 (10), 57 (88), 41 (26). - $\mathrm{C}_{22} \mathrm{H}_{34} \mathrm{O}_{3}$ (346.5). - ESI-HRMS: calcd for $\left[\mathrm{M}+\mathrm{H}^{+}\right] 347.2581$ (correct HRMS).

trans-6: IR (film): $v_{\max } 2974,2936,2874,1718,1653,1457,1437,1391,1368,1249,1196$, 1153, 1048, 957, 903, 845, $754 \mathrm{~cm}^{-1} .{ }^{1} \mathrm{H}$ NMR $\left(250 \mathrm{MHz}, \mathrm{C}_{6} \mathrm{D}_{6}\right): \delta 0.78\left(\mathrm{~s}, 3 \mathrm{H}, \mathrm{CH}_{3}\right)$, 
0.80-1.00 (m, $2 \mathrm{H}), 1.09$ [s, $\left.9 \mathrm{H}, \mathrm{C}\left(\mathrm{CH}_{3}\right)_{3}\right], 1.13-1.38(\mathrm{~m}, 3 \mathrm{H}), 1.52-1.79(\mathrm{~m}, 3 \mathrm{H}), 1.82-2.27$ (m, $4 \mathrm{H}), 2.30-2.58(\mathrm{~m}, 6 \mathrm{H}), 2.61\left(\mathrm{~s}, 3 \mathrm{H}, \mathrm{NCH}_{3}\right), 2.99\left(\mathrm{t},{ }^{3} J=13.6 \mathrm{~Hz}, 1 \mathrm{H}\right), 3.39\left(\mathrm{t},{ }^{3} J=\right.$ $8.3 \mathrm{~Hz}, 1 \mathrm{H}, 10-\mathrm{H}), 3.52-3.68\left(\mathrm{~m}, 4 \mathrm{H}, \mathrm{OCH}_{2} \mathrm{CH}_{2} \mathrm{O}\right) .-{ }^{13} \mathrm{C} \mathrm{NMR}\left(62.9 \mathrm{MHz}, \mathrm{C}_{6} \mathrm{D}_{6}\right.$, add. DEPT): ठ $11.3\left(+, \mathrm{CH}_{3}\right), 23.9\left(-, \mathrm{CH}_{2}\right), 24.3\left(-, \mathrm{CH}_{2}\right), 24.8(+, \mathrm{CH}), 25.3\left(-, \mathrm{CH}_{2}\right), 28.9[+, \mathrm{CH}, 3 \mathrm{C}$, $\left.\mathrm{C}\left(\mathrm{CH}_{3}\right)_{3}\right], 31.7\left(-, \mathrm{CH}_{2}\right), 33.9\left(-, \mathrm{CH}_{2}\right), 34.5(+, \mathrm{CH}), 34.5\left(-, \mathrm{CH}_{2}\right), 40.4\left(-, \mathrm{CH}_{2}\right), 41.6(+, \mathrm{CH})$, $42.3(+, \mathrm{CH}), 42.8(+, \mathrm{CH}), 42.8\left(\mathrm{C}_{\text {quat }}, \mathrm{C}-13\right), 44.0(+, \mathrm{CH}), 64.1\left(-, 2 \mathrm{C}, \mathrm{CH}_{2}, \mathrm{OCH}_{2} \mathrm{CH}_{2} \mathrm{O}\right)$, $72.2\left[\mathrm{C}_{\text {quat }}, C\left(\mathrm{CH}_{3}\right)_{3}\right], 80.8(+, \mathrm{CH}, \mathrm{C}-17), 110.0\left(\mathrm{C}_{\text {quat }}, \mathrm{C}-5\right), 130.1\left(\mathrm{C}_{\text {quat }}\right), 131.4\left(\mathrm{C}_{\text {quat }}\right), 177.4$ $\left(\mathrm{C}_{\text {quat }}, \mathrm{NC}=\mathrm{O}\right), 177.7\left(\mathrm{C}_{\text {quat }}, \mathrm{NC}=\mathrm{O}\right) .-\mathrm{MS}(70 \mathrm{eV}), \mathrm{m} / z(\%): 457(2)\left[\mathrm{M}^{+\bullet}\right], 401(20), 400(6)$, 356 (2), 339 (8), 289 (2), 227 (1), 155 (1), 112 (2), 99 (7), 78 (100), 52 (10), 45 (5). - $\mathrm{C}_{27} \mathrm{H}_{39} \mathrm{O}_{5} \mathrm{~N}$ (457.6): - ESI-HRMS: calcd for $\left[\mathrm{M}+\mathrm{H}^{+}\right] 458.2901$ (correct mass).

trans-7: $[\alpha]_{\mathrm{D}}^{20}=+79.5,(c=1.17, \mathrm{MeOAc}) .-\mathrm{IR}($ film $): v_{\max } 2972,2845,1739,1652,1635$, $1464,1419,1387,1374,1250,1224,1191,1109,1090,1014,923,858 \mathrm{~cm}^{-1} .-{ }^{1} \mathrm{H}$ NMR (300 MHz, $\left.\mathrm{CDCl}_{3}\right)$ : $\delta 0.92\left(\mathrm{~s}, 3 \mathrm{H}, \mathrm{CH}_{3}\right), 1.09\left[\mathrm{~s}, 9 \mathrm{H}, \mathrm{C}\left(\mathrm{CH}_{3}\right)_{3}\right], 1.13-1.60(\mathrm{~m}, 3 \mathrm{H}), 1.65-1.99$ (m, $5 \mathrm{H}), 2.42-2.76(\mathrm{~m}, 7 \mathrm{H}), 3.00-3.11(\mathrm{~m}, 1 \mathrm{H}), 3.19-3.25(\mathrm{~m}, 1 \mathrm{H}), 3.31\left(\mathrm{dd},{ }^{3} J=8.7,{ }^{3} J=\right.$ $6.3 \mathrm{~Hz}, 1 \mathrm{H}, 17-\mathrm{H}), 3.72\left(\mathrm{~s}, 3 \mathrm{H}, \mathrm{OCH}_{3}\right), 3.77$ (s, $\left.3 \mathrm{H}, \mathrm{OCH}_{3}\right), 3.91-4.05\left(\mathrm{~m}, 4 \mathrm{H}, \mathrm{OCH}_{2} \mathrm{CH}_{2} \mathrm{O}\right)$. $-{ }^{13} \mathrm{C}$ NMR (75.6 MHz, $\mathrm{CDCl}_{3}$, add. APT): $\delta 11.7\left(+, \mathrm{CH}_{3}\right), 23.4\left(-, \mathrm{CH}_{2}\right), 24.8\left(-, \mathrm{CH}_{2}\right), 26.1$ (-, $\left.\mathrm{CH}_{2}\right), 28.7\left[+, 3 \mathrm{C}, \mathrm{C}\left(\mathrm{CH}_{3}\right)_{3}\right], 31.3\left(-, \mathrm{CH}_{2}\right), 37.4\left(-, \mathrm{CH}_{2}\right), 38.7(+, \mathrm{CH}), 39.3\left(-, \mathrm{CH}_{2}\right), 40.3$ $(+, \mathrm{CH}), 43.8\left(-, \mathrm{CH}_{2}\right), 44.1\left(-, \mathrm{C}_{\text {quat }}, \mathrm{C}-13\right), 52.0(+, \mathrm{CH}), 52.1(+, \mathrm{CH}), 54.4(+, \mathrm{CH}), 64.4(-$, $\left.\mathrm{CH}_{2}, \mathrm{OCH}_{2} \mathrm{CH}_{2} \mathrm{O}\right), 64.4\left(-, \mathrm{CH}_{2}, \mathrm{OCH}_{2} \mathrm{CH}_{2} \mathrm{O}\right), 72.4$ [-, $\left.\mathrm{C}_{\text {quat }}, \mathrm{C}\left(\mathrm{CH}_{3}\right)_{3}\right], 79.6(+, \mathrm{CH}, \mathrm{C}-17)$, 108.1 (-, $\left.\mathrm{C}_{\text {quat }}, \mathrm{C}-3\right), 126.3$ (-, $\left.\mathrm{C}_{\text {quat }}\right), 130.8$ (-, $\left.\mathrm{C}_{\text {quat }}\right), 135.6\left(-, \mathrm{C}_{\text {quat }}\right), 149.0$ (-, $\left.\mathrm{C}_{\text {quat }}\right), 168.4$ (-, $\left.\mathrm{C}_{\text {quat }}, \mathrm{C}=\mathrm{O}\right), 169.0\left(-, \mathrm{C}_{\text {quat }}, \mathrm{C}=\mathrm{O}\right) .-\mathrm{MS}(70 \mathrm{eV}), \mathrm{m} / z(\%): 488(6)\left[\mathrm{M}^{+*}\right], 456(100), 428(3), 399$ (54), 341 (6), 301 (5), 288 (9), 275 (12), 230 (4), 203 (1), 143 (2), 115 (4), 99 (15), 86 (12), 57 (52), 41 (12). $-\mathrm{C}_{28} \mathrm{H}_{40} \mathrm{O}_{7}$ (488.6). - ESI-HRMS: calcd for $\left[\mathrm{M}+\mathrm{H}^{+}\right] 489.2847$ (correct HRMS).

trans-8: IR (film): $v_{\max } 2973,2931,1717,1675,1635,1617,1472,1457,1437,1419,1389$, 1363, 1249, 1198, 1142, 1118, 1092, 1066, 983, 944, 912, 883, $862 \mathrm{~cm}^{-1} .-{ }^{1} \mathrm{H}$ NMR (250 MHz, $\mathrm{CDCl}_{3}$ ): (distinguishable signals due to a single isomer are marked with "\#) $\delta 0.86$ (s, $3 \mathrm{H}, \mathrm{CH}_{3}$ ), $0.86\left(\mathrm{~s}, 3 \mathrm{H}, \mathrm{CH}_{3}\right)^{\#}, 1.15\left[\mathrm{~s}, 9 \mathrm{H}, \mathrm{C}\left(\mathrm{CH}_{3}\right)_{3}\right], 1.17\left[\mathrm{~s}, 9 \mathrm{H}, \mathrm{C}\left(\mathrm{CH}_{3}\right)_{3}\right]^{\#}, 1.22-1.63(\mathrm{~m}, 11 \mathrm{H})$, 1.71-2.82 (m, 8 H), 3.38-3.47 (m, $1 \mathrm{H}), 3.99\left(\mathrm{~m}_{\mathrm{c}}, 4 \mathrm{H}, \mathrm{OCH}_{2} \mathrm{CH}_{2} \mathrm{O}\right) .-{ }^{13} \mathrm{C}$ NMR $(75.5 \mathrm{MHz}$, $\mathrm{CDCl}_{3}$, add. APT): $\delta 11.3\left(+, \mathrm{CH}_{3}\right), 11.4\left(+, \mathrm{CH}_{3}\right), 24.8\left(-, \mathrm{CH}_{2}\right), 25.1\left(-, \mathrm{CH}_{2}\right), 25.6\left(-, \mathrm{CH}_{2}\right)$, 
$25.7\left(-, \mathrm{CH}_{2}\right), 25.9\left(-, \mathrm{CH}_{2}\right), 26.8\left(-, \mathrm{CH}_{2}\right), 28.7\left[+, 3 \mathrm{C}, \mathrm{C}\left(\mathrm{CH}_{3}\right)_{3}\right], 29.2\left(-, \mathrm{CH}_{2}\right), 31.1\left(-, \mathrm{CH}_{2}\right)$, $31.2\left(-, \mathrm{CH}_{2}\right), 34.5(+, \mathrm{CH}), 35.1\left(-, \mathrm{CH}_{2}\right), 35.5\left(-, \mathrm{CH}_{2}\right), 35.7\left(-, \mathrm{CH}_{2}\right), 37.4\left(-, \mathrm{CH}_{2}\right), 40.8(-$, $\left.\mathrm{CH}_{2}\right), 41.4\left(-, \mathrm{CH}_{2}\right), 41.6\left(-, \mathrm{CH}_{2}\right), 41.8\left(-, \mathrm{CH}_{2}\right), 42.7\left(-, \mathrm{C}_{\text {quat }}\right), 43.4\left(-, \mathrm{C}_{\text {quat }}\right), 45.6(+, \mathrm{CH})$, $45.8(+, \mathrm{CH}), 46.7(+, \mathrm{CH}), 47.7(+, \mathrm{CH}), 58.5\left(-, \mathrm{C}_{\text {quat }}\right), 59.0\left(-, \mathrm{C}_{\text {quat }}\right), 60.4\left(-, \mathrm{C}_{\text {quat }}\right), 64.4(-$, $\left.\mathrm{CH}_{2}, \mathrm{OCH}_{2} \mathrm{CH}_{2} \mathrm{O}\right), 72.4$ [-, $\left.\mathrm{C}_{\text {quat }}, \mathrm{C}\left(\mathrm{CH}_{3}\right)_{3}\right], 79.9(+, \mathrm{CH}, \mathrm{C}-17), 80.0(+, \mathrm{CH}, \mathrm{C}-17), 108.3(-$, $\left.\mathrm{C}_{\text {quat, }} \mathrm{C}-3\right), 108.4$ (-, $\left.\mathrm{C}_{\text {quat, }}, \mathrm{C}-3\right), 119.5$ (-, $\left.\mathrm{C}_{\text {quat }}\right), 120.7$ (-, $\left.\mathrm{C}_{\text {quat }}\right), 127.4$ (-, $\left.\mathrm{C}_{\text {quat }}\right), 128.3$ (-, $\left.\mathrm{C}_{\text {quat }}\right)$, 129.2 (-, $\left.\mathrm{C}_{\text {quat }}\right), 129.5$ (-, $\left.\mathrm{C}_{\text {quat }}\right), 129.7$ (-, $\left.\mathrm{C}_{\text {quat }}\right) .-\mathrm{MS}(70 \mathrm{eV}), \mathrm{m} / \mathrm{z}(\%): 435 / 433(1 / 4)\left[\mathrm{M}^{+*}\right]$, 397 (1), 379/377 (4/11), 341 (8), 315 (2), 289/287 (3/10), 252 (36), 246 (15), 208 (6), 166 (2), 155 (100), 133 (8), 111 (14), 96 (21), 84 (25), 57 (27), 41 (9). - $\mathrm{C}_{25} \mathrm{H}_{36} \mathrm{ClNO}_{3}$ (434.0). - ESIHRMS: calcd for $\left[\mathrm{M}+\mathrm{H}^{+}\right] 434.2457$ (correct mass).

trans-9: $[\alpha]_{\mathrm{D}}^{20}=+60.3,(c=1.09, \mathrm{MeOAc}) .-\mathrm{IR}\left(\right.$ film): $v_{\max } 30672973,2931,1768,1714,1685$, 1646, 1559, 1503, 1457, 1419, 1362, 1299, 1267, 1244, 1194, 1143, 1092, 1073, 945, 911, $733 \mathrm{~cm}^{-1} .{ }^{1} \mathrm{H}$ NMR $\left(300 \mathrm{MHz}, \mathrm{CDCl}_{3}\right): \delta 1.02\left(\mathrm{~s}, 3 \mathrm{H}, \mathrm{CH}_{3}\right), 1.10\left[\mathrm{~s}, 9 \mathrm{H}, \mathrm{C}\left(\mathrm{CH}_{3}\right)_{3}\right], 1.42-1.73$ $(\mathrm{m}, 6 \mathrm{H}), 1.79-2.21(\mathrm{~m}, 7 \mathrm{H}), 2.77\left(\mathrm{~m}_{\mathrm{C}}, 2 \mathrm{H}\right), 3.08\left(\mathrm{~m}_{\mathrm{c}}, 1 \mathrm{H}\right), 3.39\left(\mathrm{t},{ }^{3} J=7.3,1 \mathrm{H}, 17-\mathrm{H}\right)$, 3.90-4.08 (m, $5 \mathrm{H}), 7.27-7.53(\mathrm{~m}, 5 \mathrm{H}, \mathrm{Ph}) .-{ }^{13} \mathrm{C} \mathrm{NMR}\left(75.5 \mathrm{MHz}, \mathrm{CDCl}_{3}\right.$, add. APT): $\delta 11.8$ $\left(+, \mathrm{CH}_{3}\right), 23.9\left(-, \mathrm{CH}_{2}\right), 24.7\left(-, \mathrm{CH}_{2}\right), 25.6\left(-, \mathrm{CH}_{2}\right), 28.8\left[+, 3 \mathrm{C}, \mathrm{C}\left(\mathrm{CH}_{3}\right)_{3}\right], 31.0\left(-, \mathrm{CH}_{2}\right), 35.9$ (-, $\left.\mathrm{CH}_{2}\right), 38.9\left(-, \mathrm{CH}_{2}\right), 41.3\left(-, \mathrm{C}_{\text {quat }}, \mathrm{C}-13\right), 45.0\left(-, \mathrm{CH}_{2}\right), 52.5(+, \mathrm{CH}), 54.8(+, \mathrm{CH}), 55.5(+$, $\mathrm{CH}), 64.5\left(-, 2 \mathrm{C}, \mathrm{CH}_{2}, \mathrm{OCH}_{2} \mathrm{CH}_{2} \mathrm{O}\right), 72.5\left[-, \mathrm{C}_{\text {quat }}, C\left(\mathrm{CH}_{3}\right)_{3}\right], 79.6(+, \mathrm{CH}, \mathrm{C}-17), 107.5$ (-, $\left.\mathrm{C}_{\text {quat, }} \mathrm{C}-5\right), 123.6$ (-, $\left.\mathrm{C}_{\text {quat }}\right), 125.3$ (+, CH), 127.6 (+, CH, 2 C, Ph), 129.2 (+, CH, $\left.2 \mathrm{C}, \mathrm{Ph}\right), 129.8$ (-, $\left.\mathrm{C}_{\text {quat }}\right), 131.4\left(-, \mathrm{C}_{\text {quat }}\right), 149.8$ (-, $\left.\mathrm{C}_{\text {quat }}, \mathrm{NC}=\mathrm{O}\right), 153.5\left(-, \mathrm{C}_{\text {quat }}, \mathrm{NC}=\mathrm{O}\right) .-\mathrm{MS}(70 \mathrm{eV}), \mathrm{m} / \mathrm{z}$ (\%): 521 (100) [ $\left.\mathrm{M}^{+*}\right], 465$ (62), 420 (5), 354 (8), 345 (2), 289 (16), 289 (2), 231 (2), 178 (9), 119 (4), 99 (21), 786 (6), 57 (21), 41 (4). $-\mathrm{C}_{30} \mathrm{H}_{39} \mathrm{~N}_{3} \mathrm{O}_{5}$ (521.7). - ESI-HRMS: calcd for [M + $\left.\mathrm{H}^{+}\right]$ 522.2963 (correct HRMS).

trans-10: $[\alpha]_{\mathrm{D}}^{20}=-13.5,(c=1.23, \mathrm{MeOAc})$. - IR (film): $v_{\max } 2973,2882,1717,1700,1653$, $1635,1557,1506,1447,1417,1388,1363,1288,1238,1197,1118,1092,1060,946,910$, $734 \mathrm{~cm}^{-1} .-{ }^{1} \mathrm{H}$ NMR $\left(300 \mathrm{MHz}, \mathrm{C}_{6} \mathrm{D}_{6}\right): \delta 0.64\left(\mathrm{~s}, 3 \mathrm{H}, \mathrm{CH}_{3}\right), 1.07\left[\mathrm{~s}, 9 \mathrm{H}, \mathrm{C}\left(\mathrm{CH}_{3}\right)_{3}\right], 1.58-1.73$ (m, $5 \mathrm{H}), 1.82-2.19(\mathrm{~m}, 10 \mathrm{H}), 2.21-2.38(\mathrm{~m}, 3 \mathrm{H}), 2.68\left(\mathrm{t},{ }^{3} J=17.5 \mathrm{~Hz}, 1 \mathrm{H}\right), 3.34\left(\mathrm{t},{ }^{3} J=\right.$ 7.2 Hz, $1 \mathrm{H}, 17-\mathrm{H}), 3.43-3.60\left(\mathrm{~m}, 4 \mathrm{H}, \mathrm{OCH}_{2} \mathrm{CH}_{2} \mathrm{O}\right) .-{ }^{13} \mathrm{C}$ NMR $\left(75.5 \mathrm{MHz}, \mathrm{C}_{6} \mathrm{D}_{6}\right.$, add. APT): $\delta$ $11.1\left(+, \mathrm{CH}_{3}\right), 23.8\left(-, \mathrm{CH}_{2}\right), 24.9\left(-, \mathrm{CH}_{2}\right), 25.1\left(-, \mathrm{CH}_{2}\right), 28.9\left[+, 3 \mathrm{C}, \mathrm{C}\left(\mathrm{CH}_{3}\right)_{3}\right], 31.5\left(-, \mathrm{CH}_{2}\right)$, 
$33.5\left(-, \mathrm{CH}_{2}\right), 33.7(+, \mathrm{CH}), 34.2\left(-, \mathrm{CH}_{2}\right), 34.9\left(-, \mathrm{CH}_{2}\right), 39.3(+, \mathrm{CH}), 42.0(+, \mathrm{CH}), 42.8(-$, $\left.\mathrm{C}_{\text {quat, }} \mathrm{C}-13\right), 43.0(+, \mathrm{CH}), 44.9(+, \mathrm{CH}), 64.3\left(-, 2 \mathrm{C}, \mathrm{OCH}_{2} \mathrm{CH}_{2} \mathrm{O}\right), 72.3\left[-, \mathrm{C}_{\text {quat }}, \mathrm{C}\left(\mathrm{CH}_{3}\right)_{3}\right], 80.4$ (+, CH, C-17), 109.4 (-, $\left.\mathrm{C}_{\text {quat }}, \mathrm{C}-5\right), 130.4\left(-, \mathrm{C}_{\text {quat }}, \mathrm{C}=\mathrm{C}\right), 131.8$ (-, $\left.\mathrm{C}_{\text {quat, }} \mathrm{C}=\mathrm{C}\right), 171.9$ (-, $\mathrm{C}_{\text {quat, }}$, $\mathrm{OC}=\mathrm{O}), 172.2\left(-, \mathrm{C}_{\text {quat, }}, \mathrm{OC}=\mathrm{O}\right) .-\mathrm{MS}(70 \mathrm{eV}), \mathrm{m} / z(\%): 444(18)\left[\mathrm{M}^{+*}\right], 416(59), 388(76), 360$ (100), 342 (30), 315 (15), 297 (56), 286 (10), 253 (12), 203 (7), 169 (6), 155 (11), 131 (15), 99 (41), 86 (64), 57 (100), 41 (24). $-\mathrm{C}_{26} \mathrm{H}_{36} \mathrm{O}_{6}$ (444.6). - ESI-HRMS: calcd for $\left[\mathrm{M}+\mathrm{H}^{+}\right] 445.2585$ (correct HRMS).

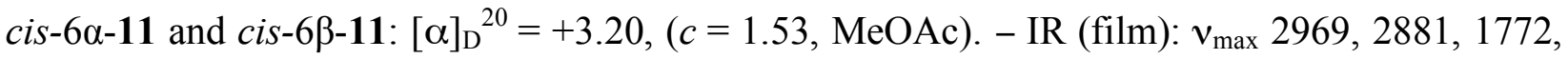
1744, 1700, 1653, 1617, 1457, 1436, 1383, 1363, 1327, 1287, 1246, 1198, 1121, 1091, 1048, 979, 948, 902, 871, $844 \mathrm{~cm}^{-1}$. ${ }^{1} \mathrm{H}$ NMR $\left(250 \mathrm{MHz}, \mathrm{CDCl}_{3}\right.$ ): (distinguishable signals due to a single isomer are marked with '\#, $) 0.83\left(\mathrm{~s}, 3 \mathrm{H}, \mathrm{CH}_{3}\right), 1.00\left(\mathrm{~s}, 3 \mathrm{H}, \mathrm{CH}_{3}\right)^{\#}, 1.16[\mathrm{~s}, 9 \mathrm{H}$, $\left.\mathrm{C}\left(\mathrm{CH}_{3}\right)_{3}\right], 1.18\left[\mathrm{~s}, 9 \mathrm{H}, \mathrm{C}\left(\mathrm{CH}_{3}\right)_{3}\right]^{\#}, 1.22-2.05(\mathrm{~m}, 7 \mathrm{H}), 2.12-2.58(\mathrm{~m}, 10 \mathrm{H}), 2.72\left(\mathrm{~m}_{\mathrm{c}}, 1 \mathrm{H}\right), 2.90$ $\left(\mathrm{s}, 3 \mathrm{H}, \mathrm{NCH}_{3}\right), 2.98\left(\mathrm{~s}, 3 \mathrm{H}, \mathrm{NCH}_{3}\right)^{\#}, 3.04\left(\mathrm{~m}_{\mathrm{C}}, 1 \mathrm{H}\right), 3.52\left(\mathrm{dd},{ }^{3} J=9.5,{ }^{3} J=8.0 \mathrm{~Hz}, 1 \mathrm{H}, 10-\mathrm{H}\right)$, 3.52-3.68 (m, $\left.4 \mathrm{H}, \mathrm{OCH}_{2} \mathrm{CH}_{2} \mathrm{O}\right) .-{ }^{13} \mathrm{C} \mathrm{NMR}\left(75.5 \mathrm{MHz}, \mathrm{CDCl}_{3}\right.$, add. APT): $\delta 22.1\left(+, \mathrm{CH}_{3}\right)$, $23.0\left(+, \mathrm{CH}_{3}\right)^{\#}, 23.4\left(-, \mathrm{CH}_{2}\right), 23.7\left(-, \mathrm{CH}_{2}\right)^{\#}, 24.5(+, \mathrm{CH}), 24.8(+, \mathrm{CH})^{\#}, 25.8\left(-, \mathrm{CH}_{2}\right), 26.0(-$, $\left.\mathrm{CH}_{2}\right)^{\#}, 26.3\left(-, \mathrm{CH}_{2}\right), 28.7\left[+, 3 \mathrm{C}, \mathrm{C}\left(\mathrm{CH}_{3}\right)_{3}\right]^{\#}, 28.8\left[+, 3 \mathrm{C}, \mathrm{C}\left(\mathrm{CH}_{3}\right)_{3}\right], 31.0\left(-, \mathrm{CH}_{2}\right), 31.6(-$, $\left.\mathrm{CH}_{2}\right)^{\#}, 33.5(+, \mathrm{CH}), 33.9\left(-, \mathrm{CH}_{2}\right), 34.1\left(-, \mathrm{CH}_{2}\right)^{\#}, 34.7\left(-, \mathrm{CH}_{2}\right), 35.0(+, \mathrm{CH})^{\#}, 35.4\left(-, \mathrm{CH}_{2}\right)$, $35.7\left(-, \mathrm{CH}_{2}\right)^{\#}, 36.6(+, \mathrm{CH}), 40.7(+, \mathrm{CH})^{\#}, 41.8(+, \mathrm{CH}), 41.9(6)(+, \mathrm{CH})^{\#}, 42.0(1)(+, \mathrm{CH})^{\#}$, $42.5\left(-, \mathrm{C}_{\text {quat }}\right), 42.9(+, \mathrm{CH}), 43.7\left(-, \mathrm{C}_{\text {quat }}\right)^{\#}, 52.9\left(+, \mathrm{NCH}_{3}\right), 64.1\left(-, \mathrm{CH}_{2}, 2 \mathrm{C}, \mathrm{OCH}_{2} \mathrm{CH}_{2} \mathrm{O}\right)$, $64.4\left(-, \mathrm{CH}_{2}, 2 \mathrm{C}, \mathrm{OCH}_{2} \mathrm{CH}_{2} \mathrm{O}\right)^{\#}, 72.3\left[-, \mathrm{C}_{\text {quat }}, \mathrm{C}\left(\mathrm{CH}_{3}\right)_{3}\right], 72.6\left[-, \mathrm{C}_{\text {quat }}, \mathrm{C}\left(\mathrm{CH}_{3}\right)_{3}\right]^{\#}, 76.2(+, \mathrm{CH}$, C-10), $80.4(+, \mathrm{CH}, \mathrm{C}-10)^{\#}, 108.2\left(-, \mathrm{C}_{\text {quat }}, \mathrm{C}-5\right), 109.9$ (-, $\left.\mathrm{C}_{\text {quat }}, \mathrm{C}-5\right)^{\#}, 125.9$ (-, $\left.\mathrm{C}_{\text {quat }}\right), 128.0$ (-, $\left.\mathrm{C}_{\text {quat }}\right)^{\#}, 129.8\left(-, \mathrm{C}_{\text {quat }}\right), 134.0\left(-, \mathrm{C}_{\text {quat }}\right)^{\#}, 177.7\left(-, \mathrm{C}_{\text {quat }}, \mathrm{C}=\mathrm{O}\right), 177.9\left(-, \mathrm{C}_{\text {quat }}, \mathrm{C}=\mathrm{O}\right), 179.7$ (-, $\left.\mathrm{C}_{\text {quat }}, \mathrm{C}=\mathrm{O}\right)^{\#}, 179.8\left(-, \mathrm{C}_{\text {quat }}, \mathrm{C}=\mathrm{O}\right)^{\#} .-\mathrm{MS}(70 \mathrm{eV}), \mathrm{m} / \mathrm{z}(\%): 457(1)\left[\mathrm{M}^{+*}\right], 412(1), 401(64), 400$ (22), 383 (10), 356 (7), 339 (22), 321 (9), 305 (4), 290 (1), 155 (2), 112 (3), 86 (5), 78 (6), 74 (26), 57 (9), 43 (100). - $\mathrm{C}_{27} \mathrm{H}_{39} \mathrm{NO}_{5}$ (457.6). - ESI-HRMS: calcd for $\left[\mathrm{M}+\mathrm{H}^{+}\right] 458.2904$ (correct mass).

trans-13: $[\alpha]_{\mathrm{D}}^{20}=-4.80,(c=1.02, \mathrm{MeOAc}) .-\mathrm{IR}$ (film): $v_{\max } 3410,2955,2878,2253,1734$, $1669,1635,1472,1457,1437,1371,1339,1254,1133,1072,1047,981,942,915,845$, $736 \mathrm{~cm}^{-1} .-{ }^{1} \mathrm{H}$ NMR $\left(300 \mathrm{MHz}, \mathrm{CDCl}_{3}\right): \delta 0.89\left(\mathrm{~s}, 3 \mathrm{H}, \mathrm{CH}_{3}\right), 1.22\left(\mathrm{~m}_{\mathrm{c}}, 1 \mathrm{H}\right), 1.35-1.71(\mathrm{~m}$, 
$3 \mathrm{H}), 1.77\left(\mathrm{~m}_{\mathrm{c}}, 1 \mathrm{H}\right), 1.90\left(\mathrm{~m}_{\mathrm{c}}, 1 \mathrm{H}\right), 2.08-2.27(\mathrm{~m}, 3 \mathrm{H}), 2.30-2.46(\mathrm{~m}, 1 \mathrm{H}), 2.49-2.60(\mathrm{~m}, 3 \mathrm{H})$, 2.72-2.93 (m, $3 \mathrm{H}), 2.97-3.08$ (m, $2 \mathrm{H}), 3.11-3.20$ (m, $2 \mathrm{H}), 3.76$ (t, $\left.{ }^{3} J=7.2 \mathrm{~Hz}, 1 \mathrm{H}, 17-\mathrm{H}\right)$.

$-{ }^{13} \mathrm{C}$ NMR (75.6 MHz, $\mathrm{CDCl}_{3}$, add. APT): $\delta 10.2\left(+, \mathrm{CH}_{3}\right), 23.2\left(-, \mathrm{CH}_{2}\right), 24.3\left(-, \mathrm{CH}_{2}\right), 27.3$ (-, $\left.\mathrm{CH}_{2}\right), 29.2\left(-, \mathrm{CH}_{2}\right), 30.3\left(-, \mathrm{CH}_{2}\right), 31.3(+, \mathrm{CH}), 35.4\left(-, \mathrm{CH}_{2}\right), 36.2(+, \mathrm{CH}), 38.3(+, \mathrm{CH})$, $40.5\left(-, \mathrm{CH}_{2}\right), 42.9$ (-, $\left.\mathrm{CH}_{2}\right), 46.9$ (+, CH), 47.4 (-, $\left.\mathrm{C}_{\text {quat }}, \mathrm{C}-13\right), 80.9$ (+, $\left.\mathrm{CH}, \mathrm{C}-17\right), 117.9$ (-, $\left.\mathrm{C}_{\text {quat }}\right), 118.6$ (-, $\left.\mathrm{C}_{\text {quat }}\right), 126.0$ (-, $\left.\mathrm{C}_{\text {quat }}\right), 129.8$ (-, $\left.\mathrm{C}_{\text {quat }}\right), 207.2\left(-, \mathrm{C}_{\text {quat }}, \mathrm{C}=\mathrm{O}\right) .-\mathrm{MS}(70 \mathrm{eV}), \mathrm{m} / \mathrm{z}$ (\%): 324 (100) $\left[\mathrm{M}^{+*}\right], 291$ (11), 265 (15), 252 (9), 226 (11), 195 (8), 129 (9), 111 (10), 84 (18), 55 (12), 41 (17). $-\mathrm{C}_{20} \mathrm{H}_{24} \mathrm{~N}_{2} \mathrm{O}_{2}$ (324.4). - ESI-HRMS: calcd for $\left[\mathrm{M}+\mathrm{H}^{+}\right] 325.1911$ (correct HRMS).

15: IR (film): $v_{\max } 3073,2972,2931,1734,1653,1635,1472,1437,1419,1388,1362,1302$, $1252,1198,1172,1109,1078,1062,1035,947,908,856 \mathrm{~cm}^{-1} .-{ }^{1} \mathrm{H}$ NMR $\left(300 \mathrm{MHz}, \mathrm{CDCl}_{3}\right): \delta$ $0.93\left(\mathrm{~s}, 3 \mathrm{H}, \mathrm{CH}_{3}\right), 1.18\left[\mathrm{~s}, 9 \mathrm{H}, \mathrm{C}\left(\mathrm{CH}_{3}\right)_{3}\right], 1.60-1.81(\mathrm{~m}, 2 \mathrm{H}), 1.86\left(\mathrm{t},{ }^{3} \mathrm{~J}=6.3 \mathrm{~Hz}, 2 \mathrm{H}\right)$, 2.30-2.40 (m, $1 \mathrm{H}), 2.43-2.97$ (m, $6 \mathrm{H}), 3.21$ (d, $\left.{ }^{3} J=17.4 \mathrm{~Hz}, 1 \mathrm{H}\right), 3.76$ (s, $3 \mathrm{H}, \mathrm{OCH}_{3}$ ), 3.82 (s, $\left.3 \mathrm{H}, \mathrm{OCH}_{3}\right), 3.84\left(\mathrm{t},{ }^{3} J=6.6 \mathrm{~Hz}, 1 \mathrm{H}, 17-\mathrm{H}\right), 3.99\left(\mathrm{~m}_{\mathrm{c}}, 4 \mathrm{H}, \mathrm{OCH}_{2} \mathrm{CH}_{2} \mathrm{O}\right), 5.58\left(\mathrm{~m}_{\mathrm{c}}, 1 \mathrm{H}, 15-\mathrm{H}\right)$. $-{ }^{13} \mathrm{C}$ NMR (75.6 MHz, $\mathrm{CDCl}_{3}$, add. APT): $\delta 16.8\left(+, \mathrm{CH}_{3}\right), 24.7\left(-, \mathrm{CH}_{2}\right), 26.1\left(-, \mathrm{CH}_{2}\right), 28.7$ $\left[+, 3 \mathrm{C}, \mathrm{C}\left(\mathrm{CH}_{3}\right)_{3}\right], 30.9\left(-, \mathrm{CH}_{2}\right), 34.6\left(-, \mathrm{CH}_{2}\right), 37.2(+, \mathrm{CH}), 39.5\left(-, \mathrm{C}_{\text {quat }}, \mathrm{C}-13\right), 46.4(-$, $\left.\mathrm{CH}_{2}\right), 52.3\left(+, \mathrm{OCH}_{3}\right), 52.4\left(+, \mathrm{OCH}_{3}\right), 64.3(7)\left(-, \mathrm{CH}_{2}, \mathrm{OCH}_{2} \mathrm{CH}_{2} \mathrm{O}\right), 64.3(9)\left(-, \mathrm{CH}_{2}\right.$, $\left.\mathrm{OCH}_{2} \mathrm{CH}_{2} \mathrm{O}\right), 72.7$ [-, $\left.\mathrm{C}_{\text {quat }}, \mathrm{C}\left(\mathrm{CH}_{3}\right)_{3}\right], 80.0(+, \mathrm{CH}, \mathrm{C}-17), 107.3\left(-, \mathrm{C}_{\text {quat, }}, \mathrm{C}-3\right), 122.5(+, \mathrm{CH}, \mathrm{C}-$ $15), 128.5$ (-, $\left.\mathrm{C}_{\text {quat }}\right), 129.1$ (-, $\left.\mathrm{C}_{\text {quat }}\right), 129.6$ (-, $\left.\mathrm{C}_{\text {quat }}\right), 131.4\left(-, \mathrm{C}_{\text {quat }}\right), 135.4\left(-, \mathrm{C}_{\text {quat }}\right), 138.3$ (-, $\left.\mathrm{C}_{\text {quat }}\right), 142.6\left(-, \mathrm{C}_{\text {quat }}\right), 168.4\left(-, \mathrm{C}_{\text {quat }}, \mathrm{C}=\mathrm{O}\right), 170.3\left(-, \mathrm{C}_{\text {quat }}, \mathrm{C}=\mathrm{O}\right) .-\mathrm{DCI}-\mathrm{MS}\left(\mathrm{NH}_{3}\right), m / z(\%)$ : $502(100)\left[\mathrm{M}+\mathrm{NH}_{4}^{+}\right], 446$ (3), 428 (1), 296 (1). $-\mathrm{C}_{28} \mathrm{H}_{36} \mathrm{O}_{7}$ (484.6). - ESI-HRMS: calcd for $\left[\mathrm{M}+\mathrm{NH}_{4}{ }^{+}\right] 502.2803$ (correct HRMS).

\section{References}

(1) Busacca, C. A.; Grossbach, D.; Campbell, S. J.; Dong, Y.; Eriksson, M. C.; Harris, R. E.; Jones, P.-J.; Kim, J.-Y.; Lorenz, J. C.; McKellop, K. B.; O'Brien, E. M.; Qiu, F.; Simpson, R. D.; Smith, L.; So, R. C.; Spinelli, E. M.; Vitous, J.; Zavattaro, C. J. Org. Chem. 2004, 69, 5187-5195. 

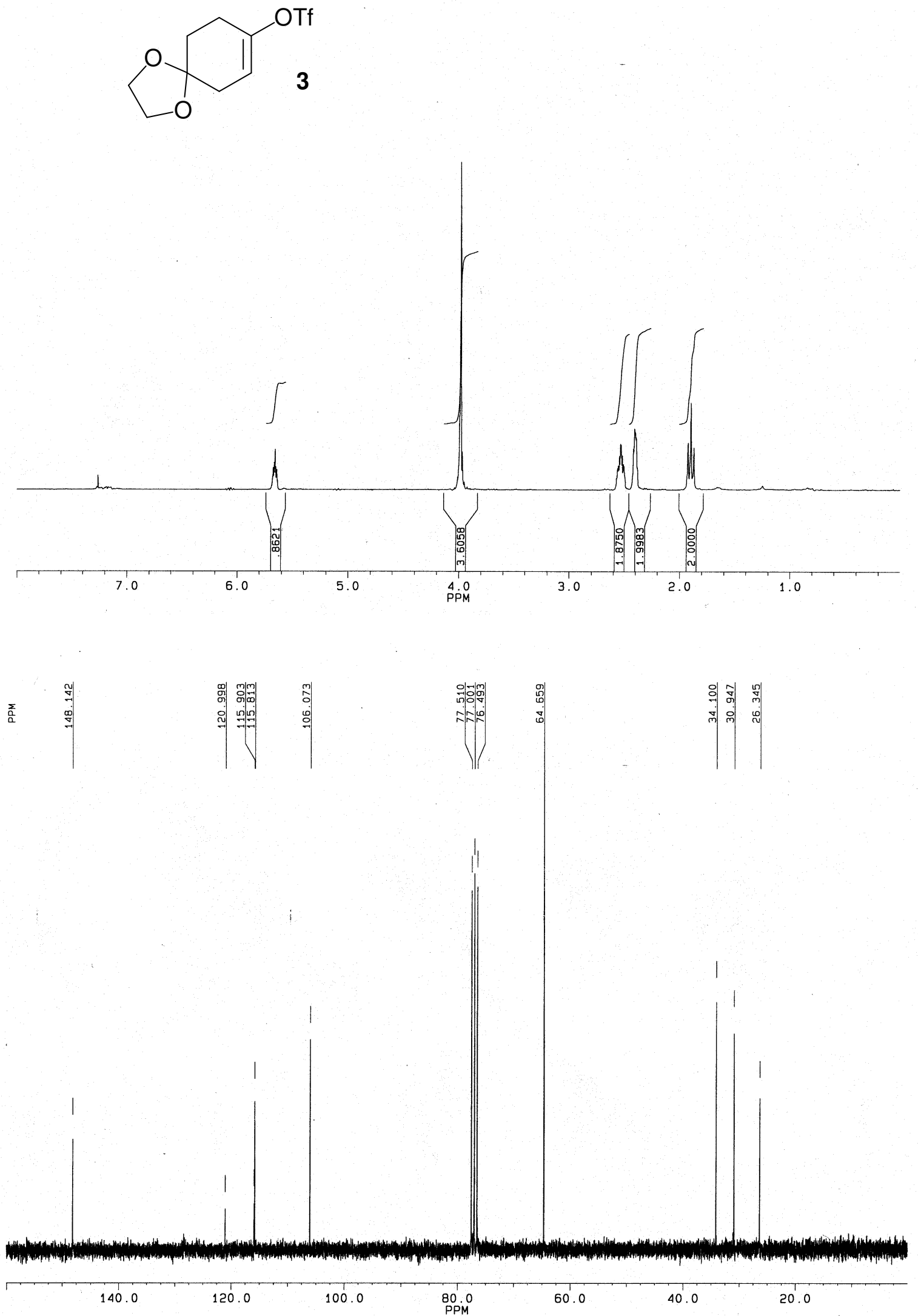
<smiles>CCCCOC1CCC2C=C(C3=CCC4(CC3)OCCO4)CC[C@@]21C</smiles>
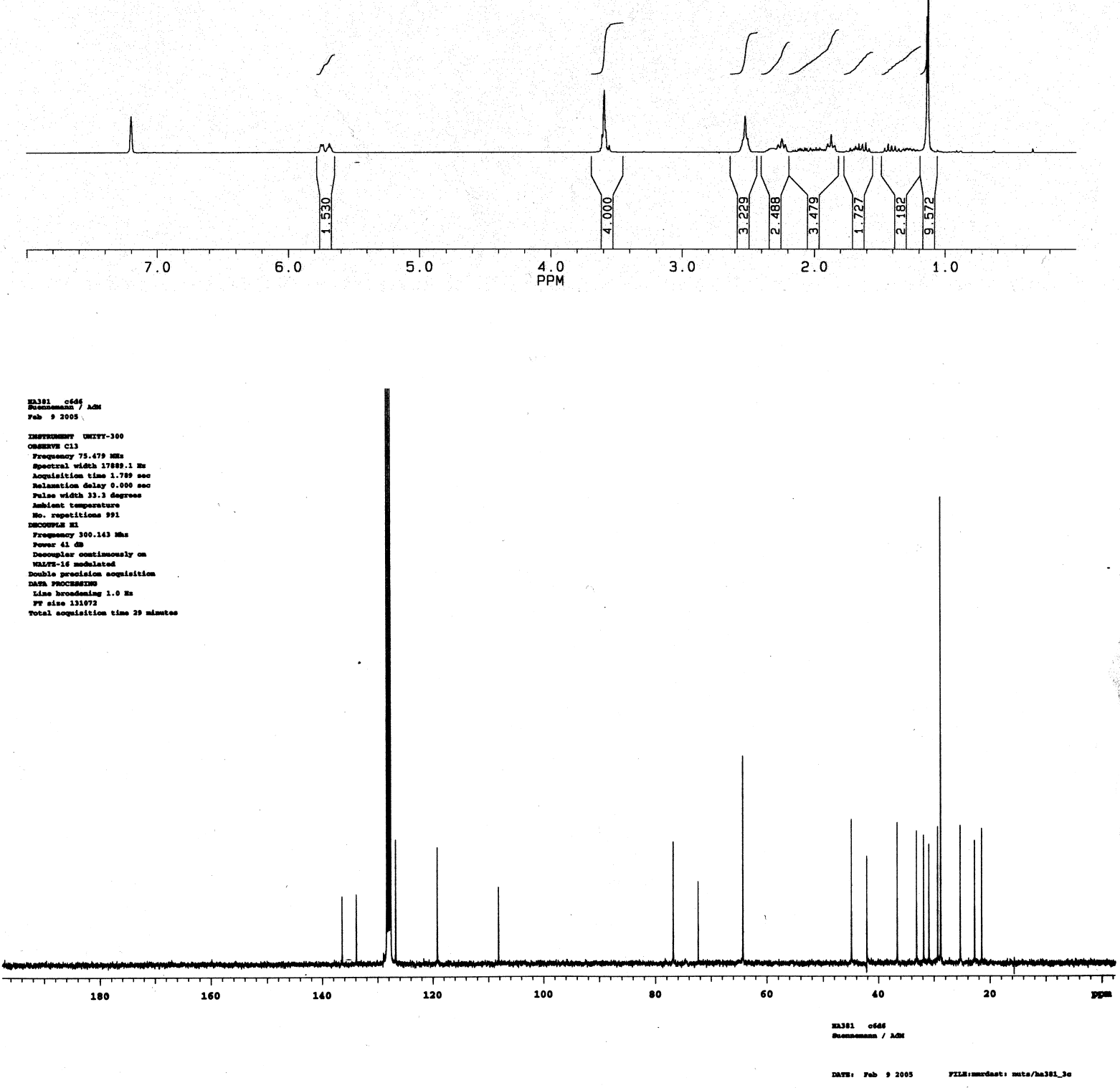

S14 


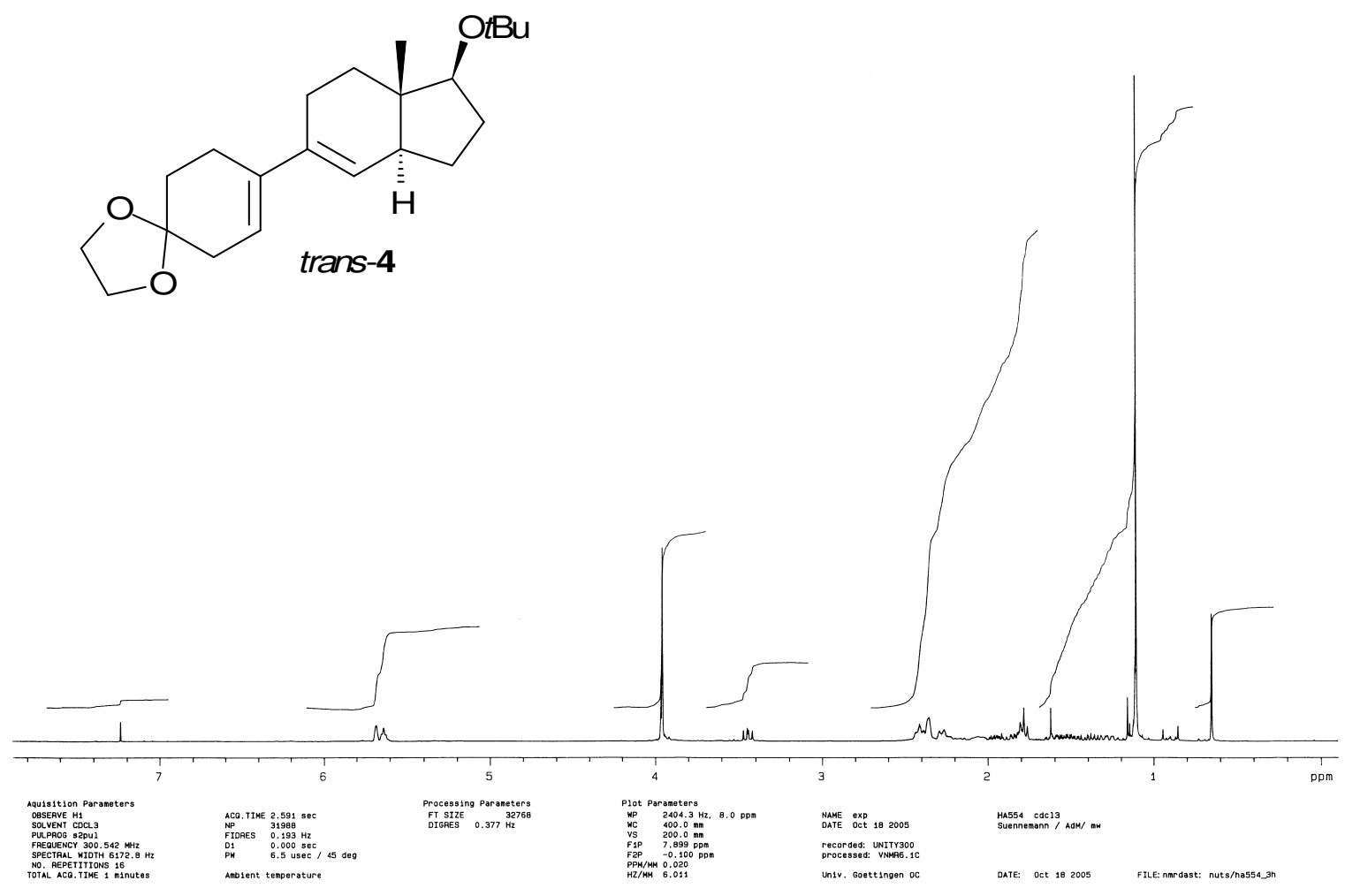

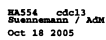

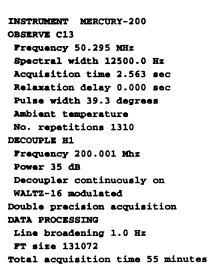

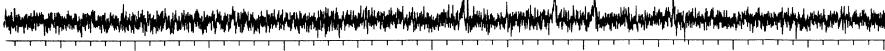

180

160

140

120

100

80

$\lim _{i \rightarrow \infty}$

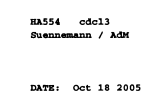



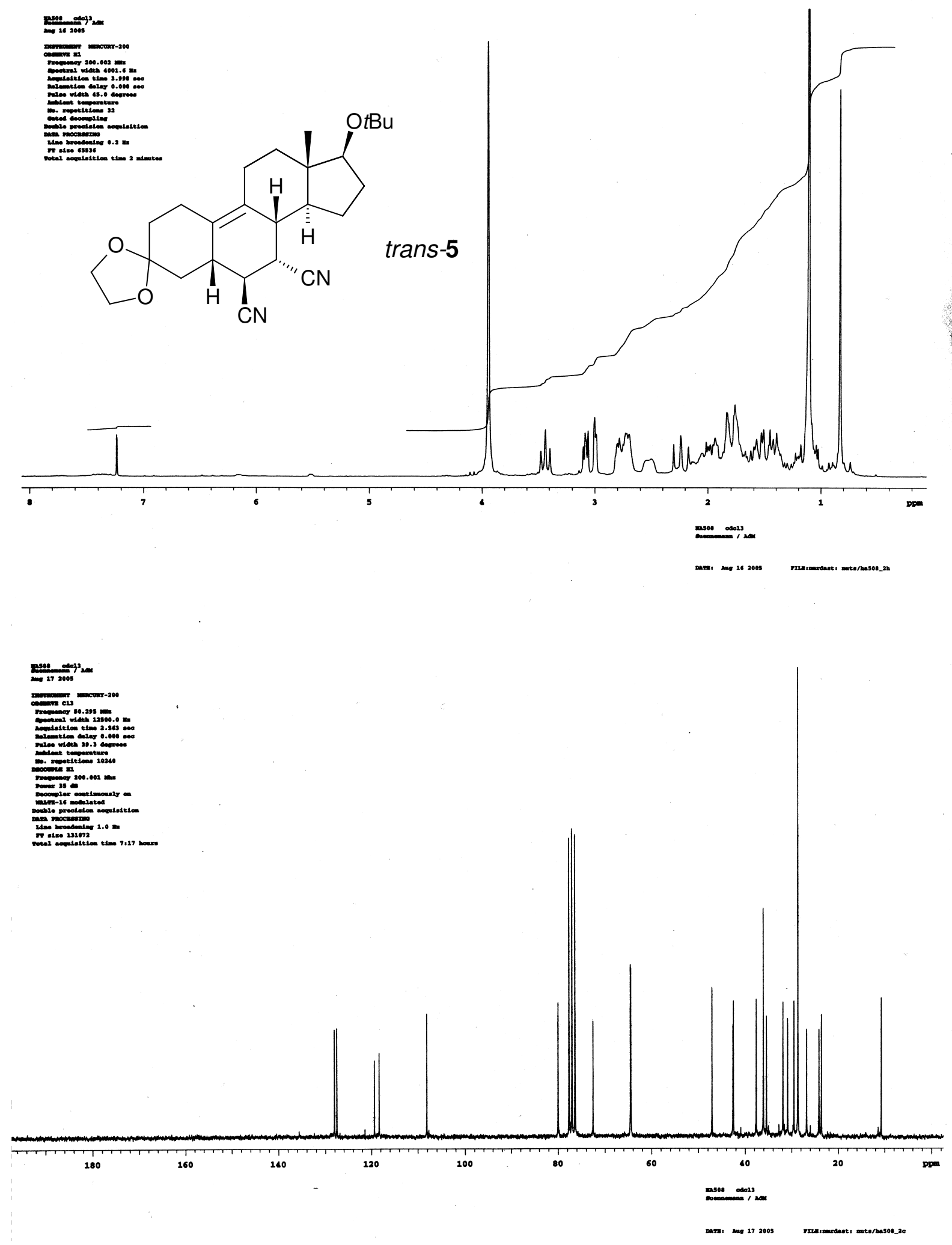

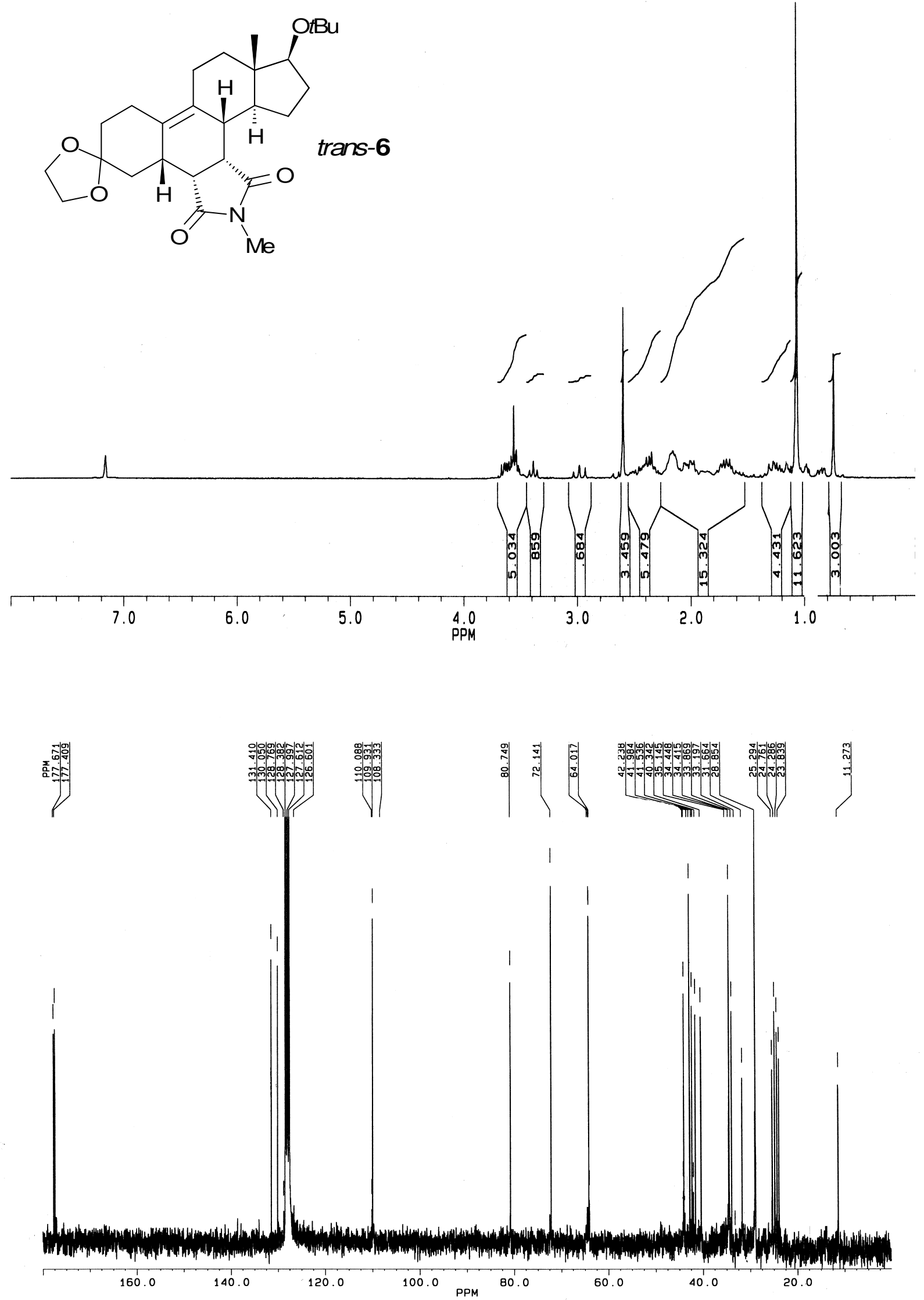


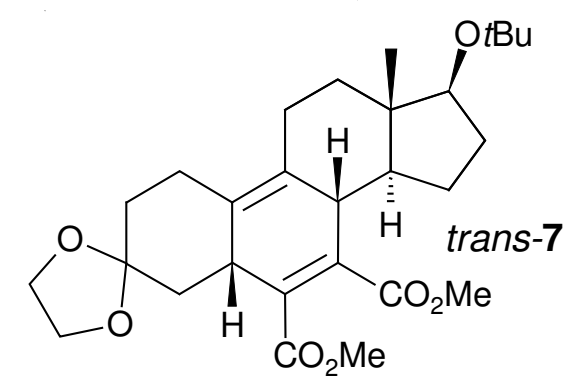

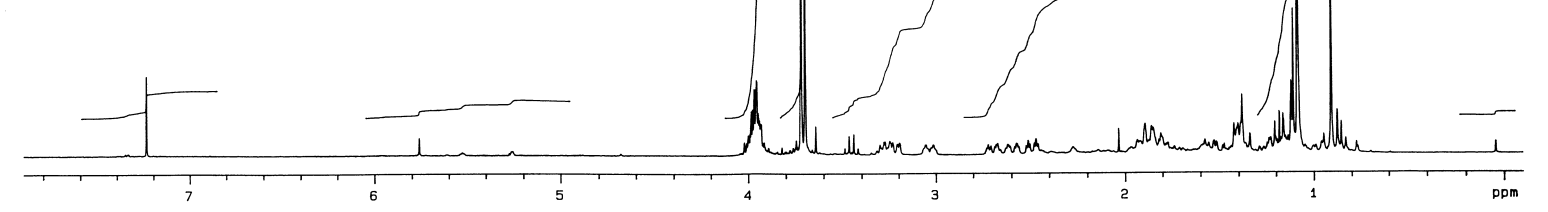

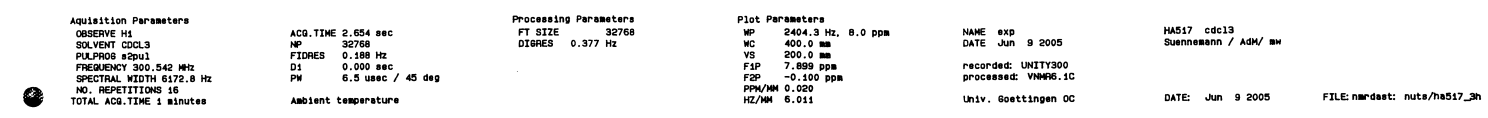
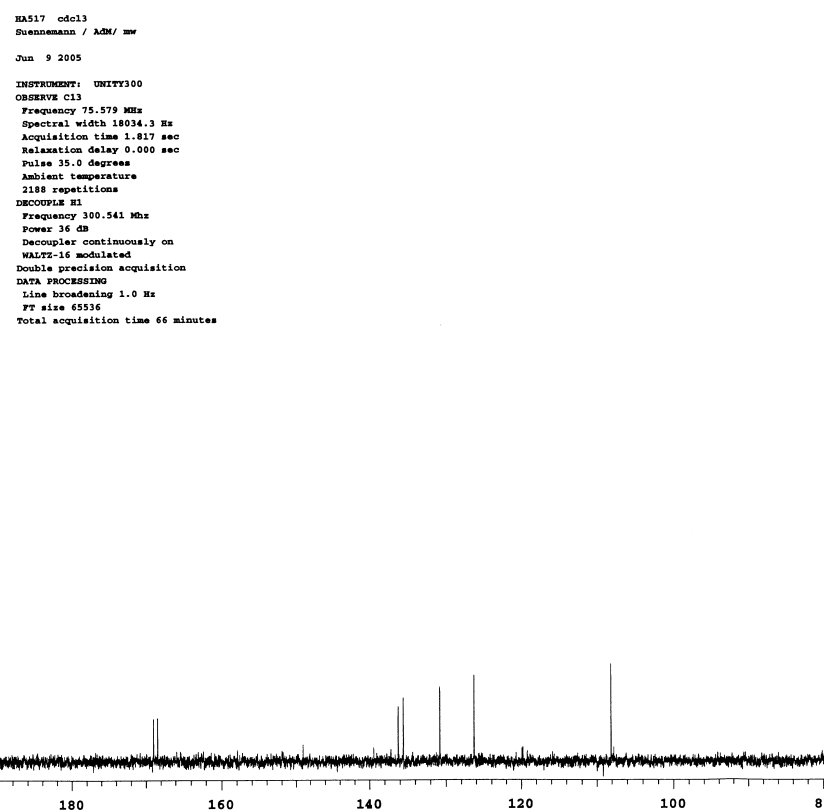

180 1 1 1 1 1 1 160

140

100

80

Darz: Tun 92005 

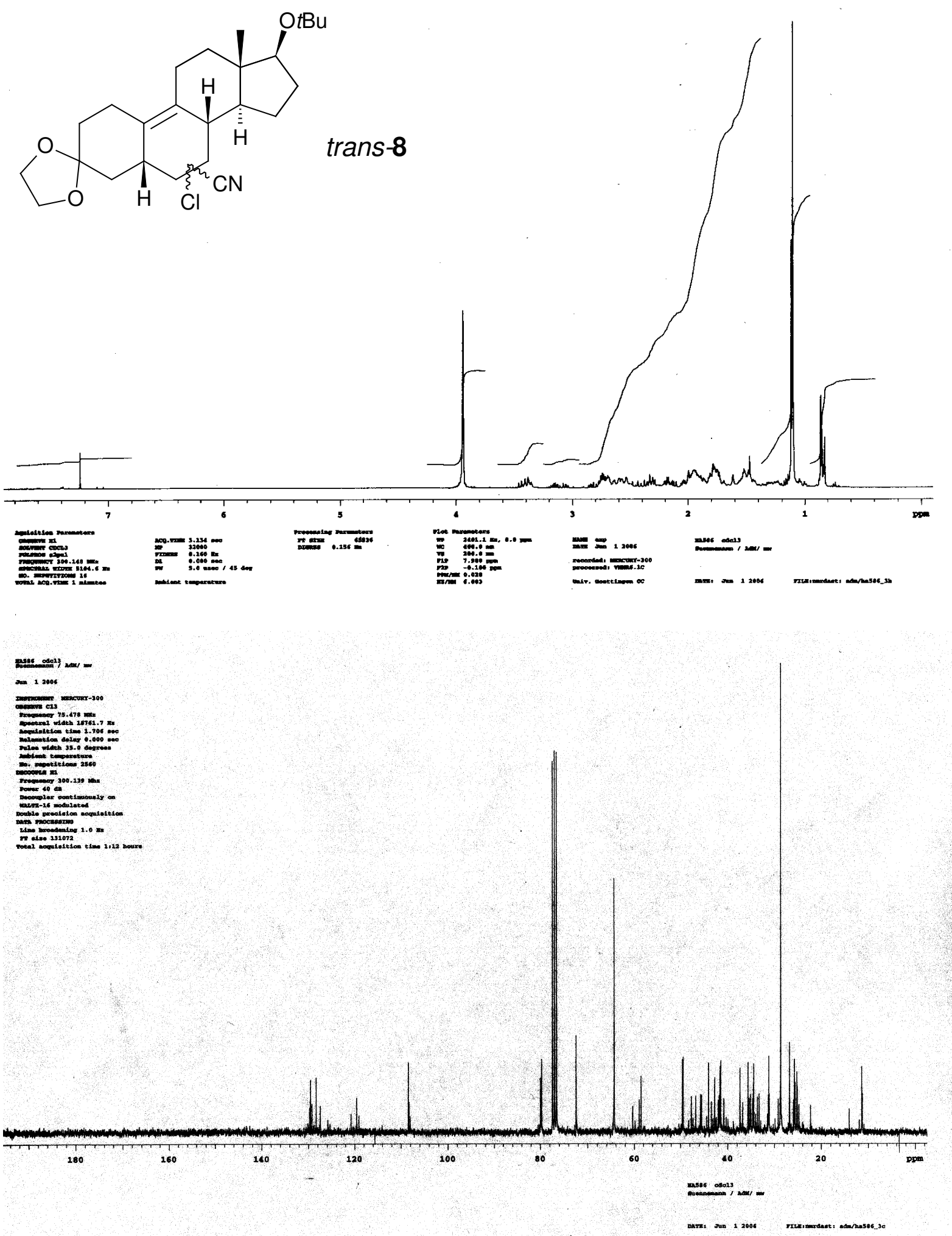

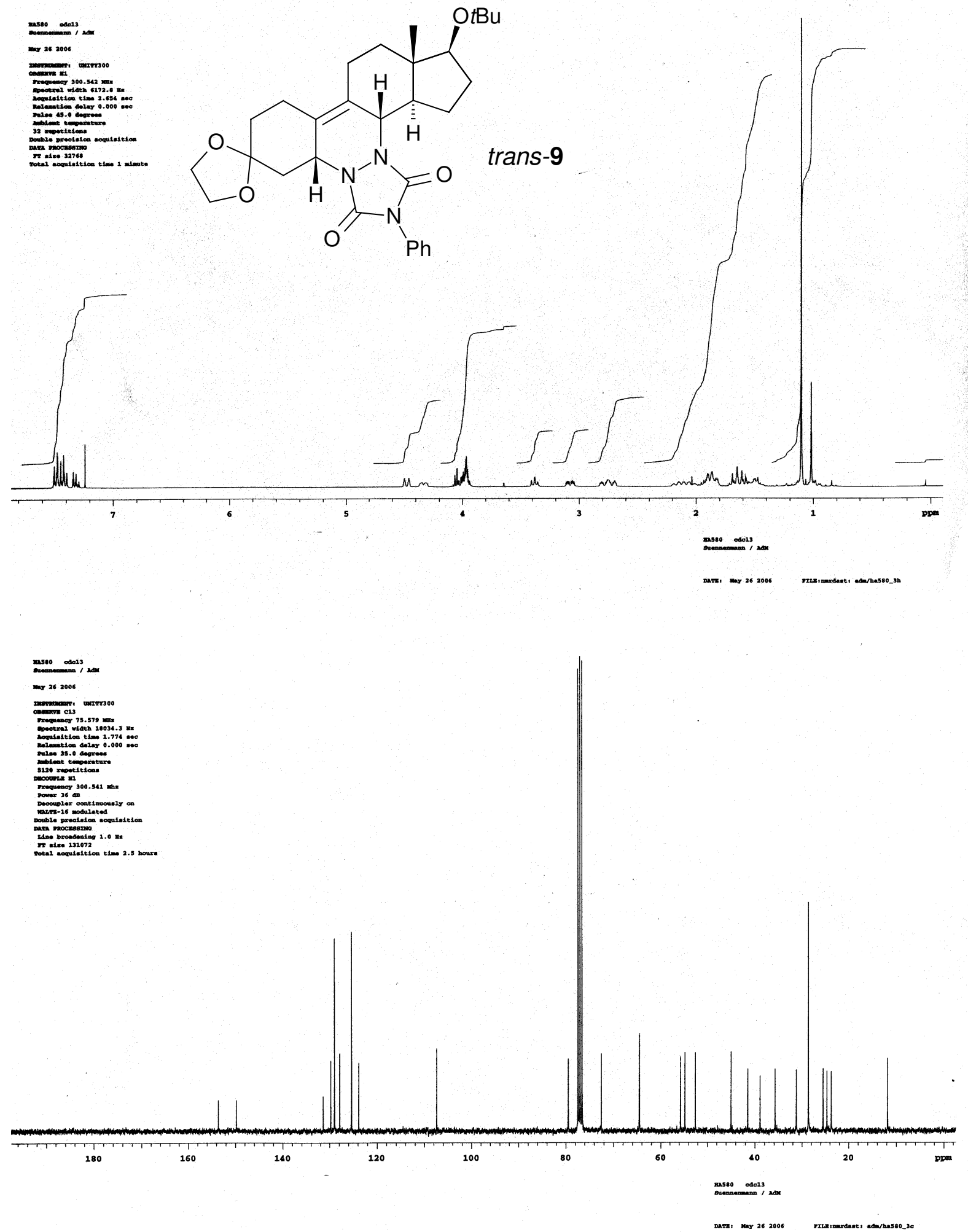


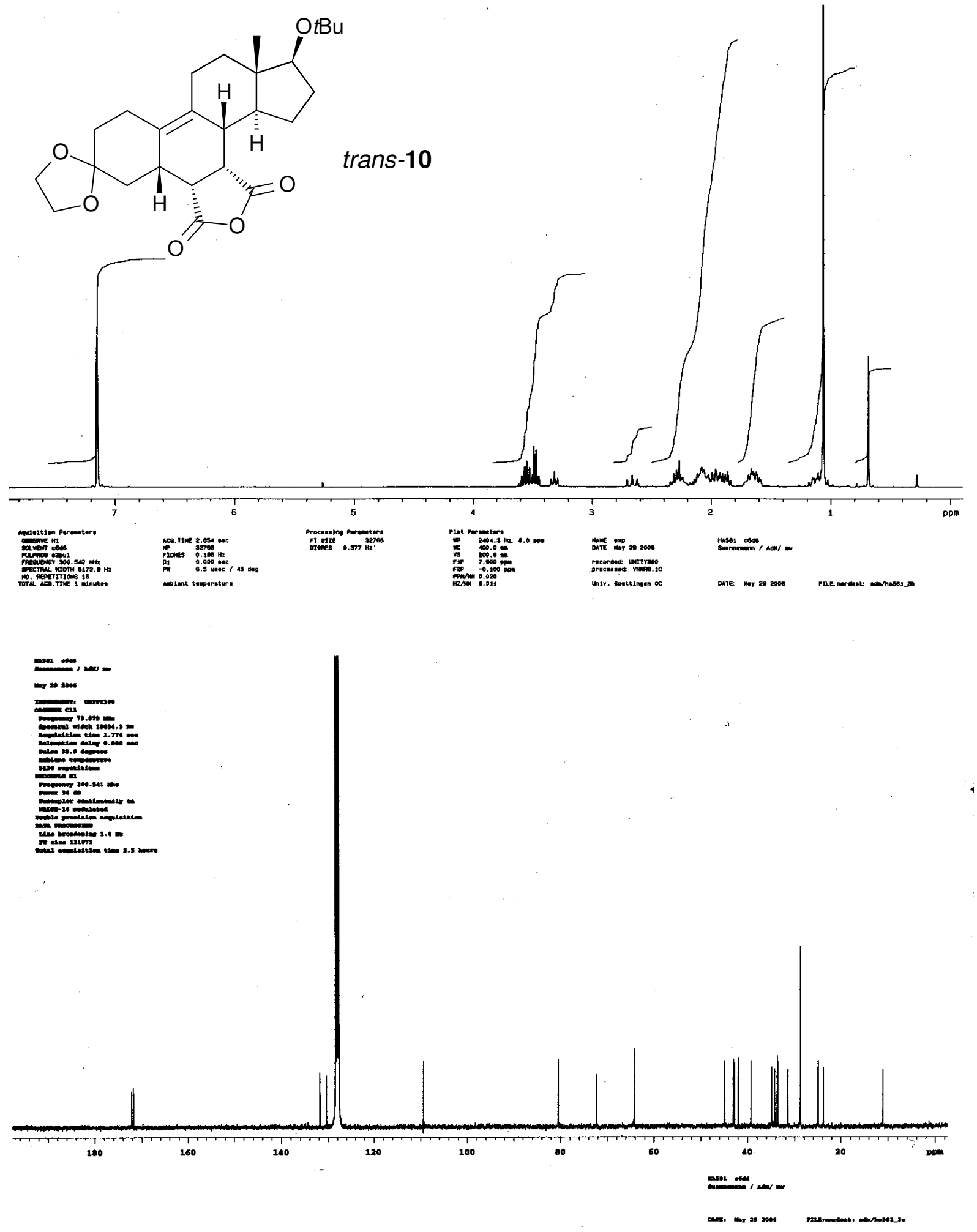




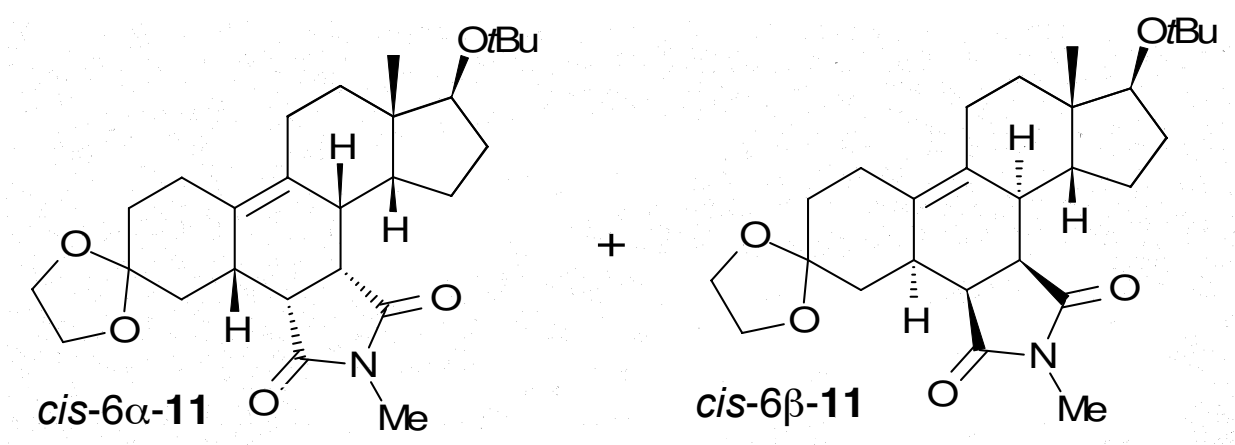
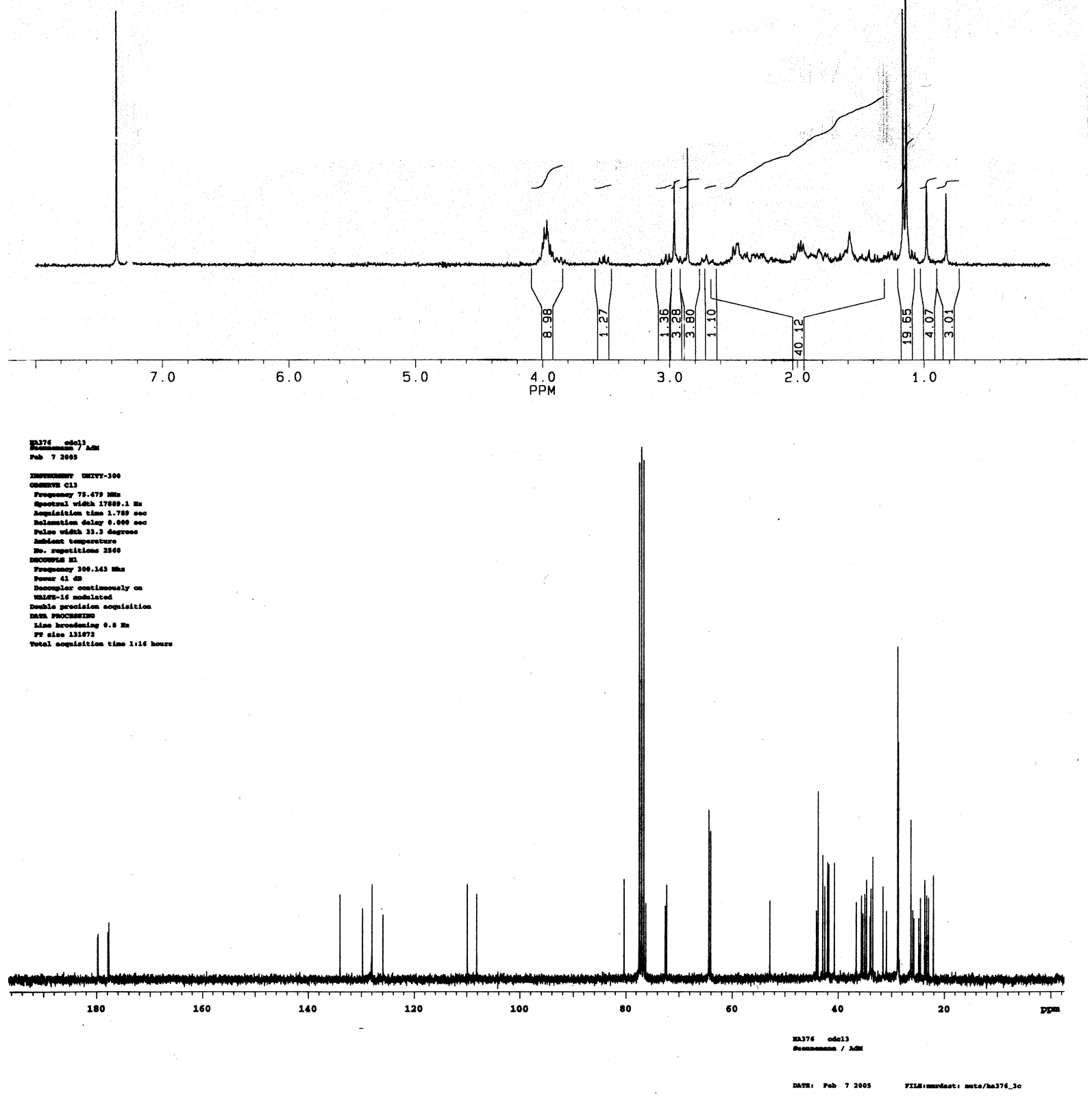


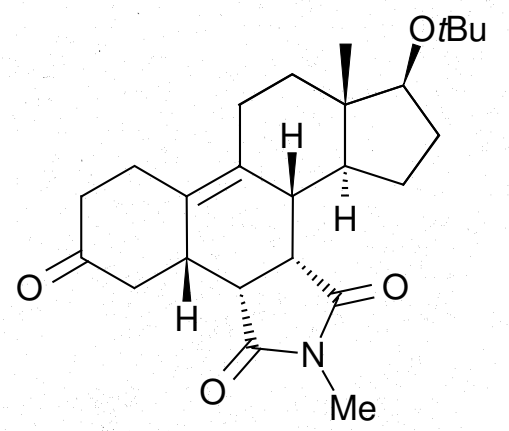

mono-deprotected derivative of trans-6
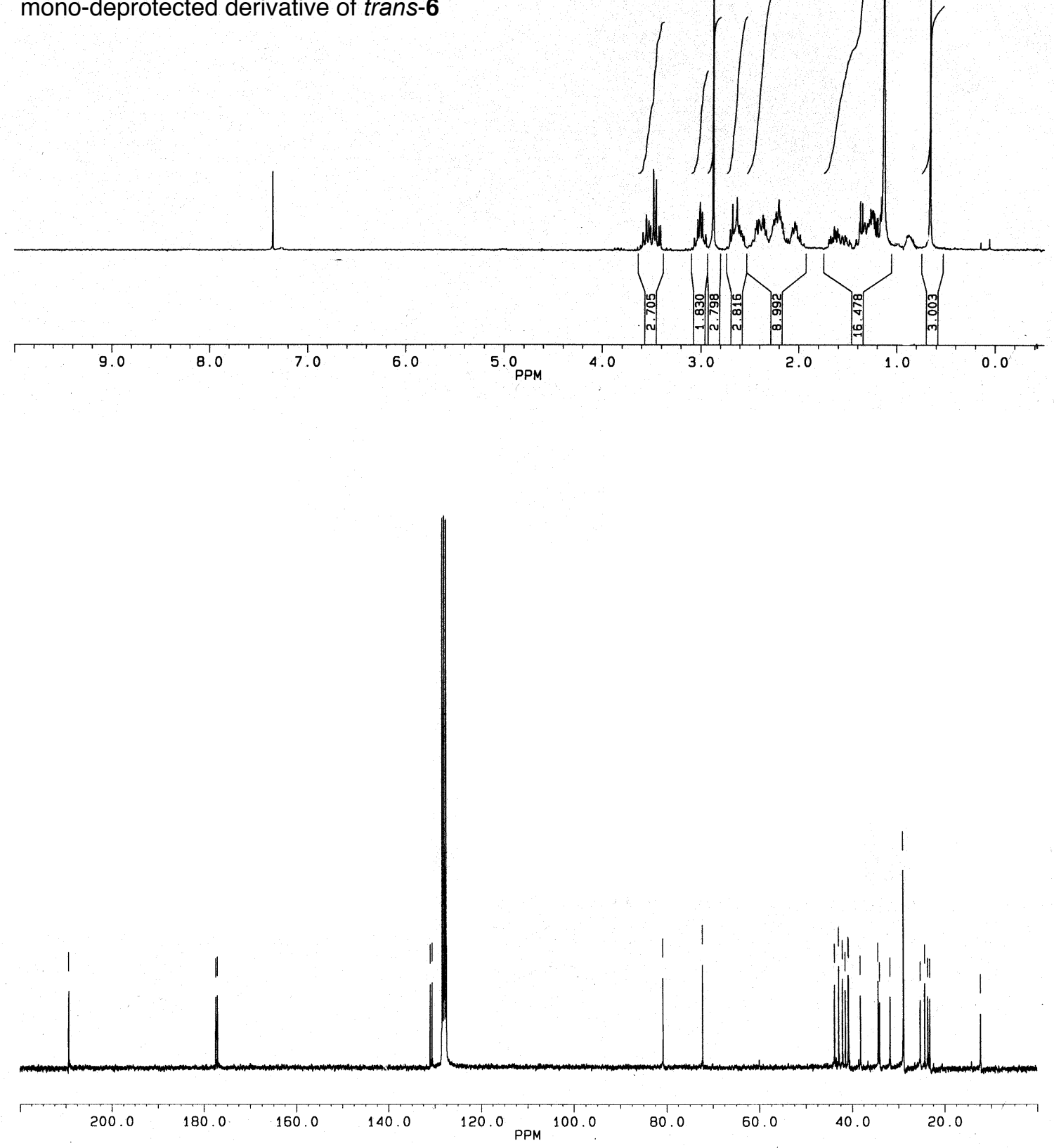

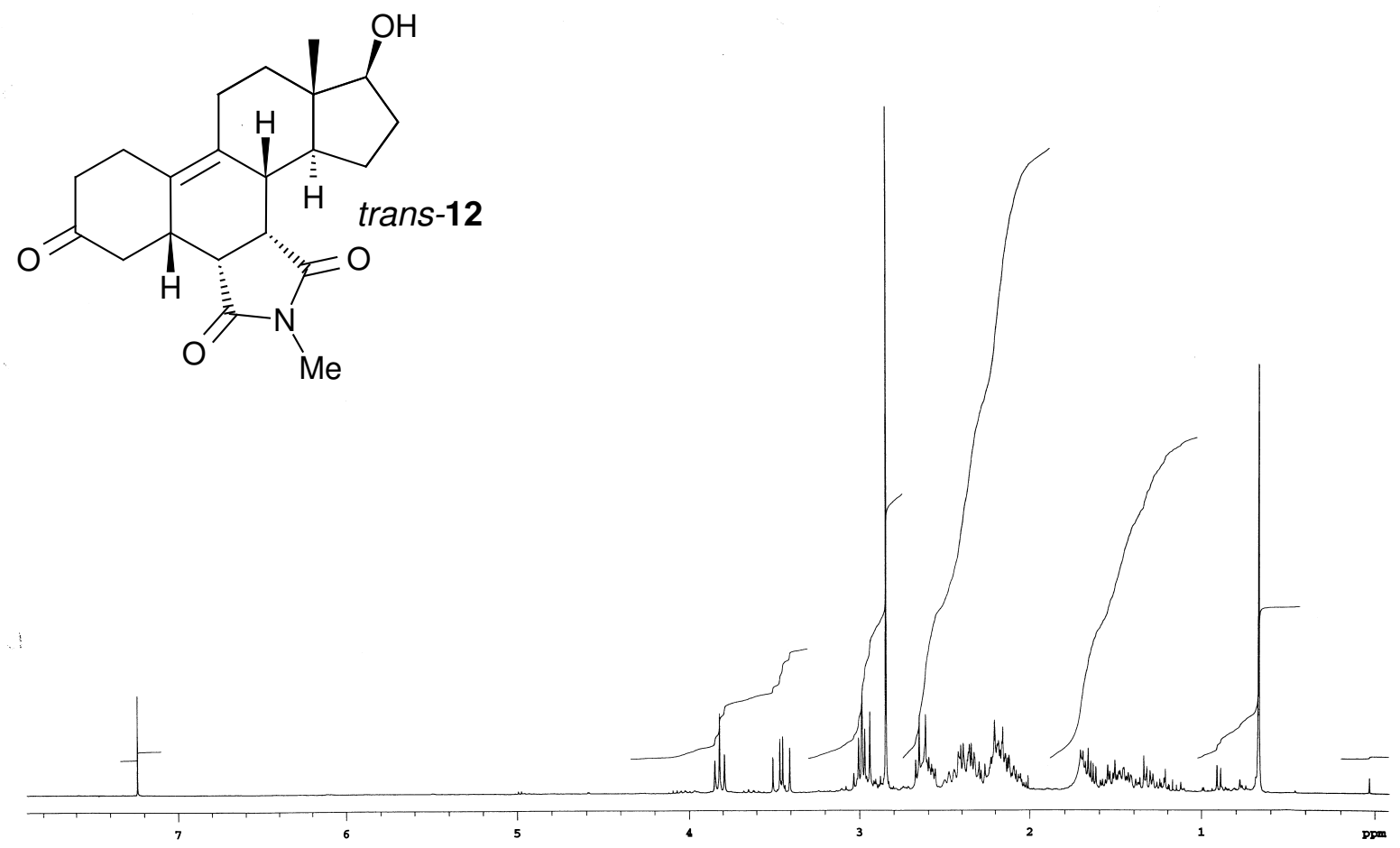

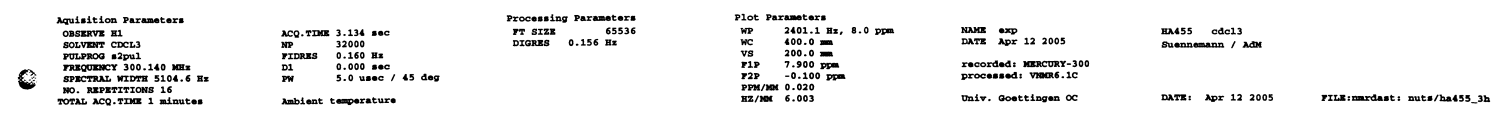

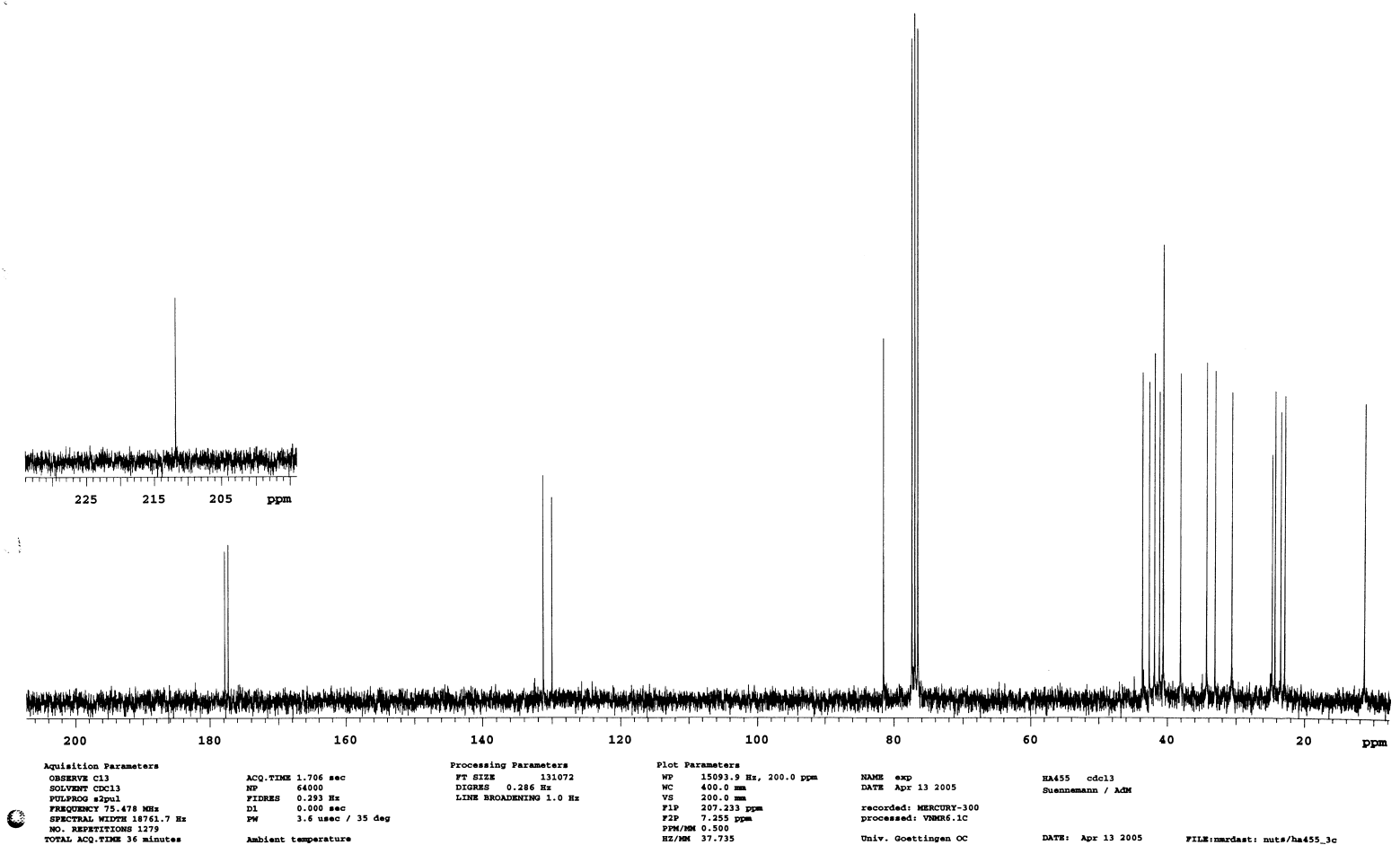


<smiles>CCCCOC1CC[C@H]2[C@H]3C(=C4CCC(=O)C[C@H]4[C@H](C#N)[C@H]3C#N)CC[C@]12C</smiles>

mono-deprotected derivative of trans-5
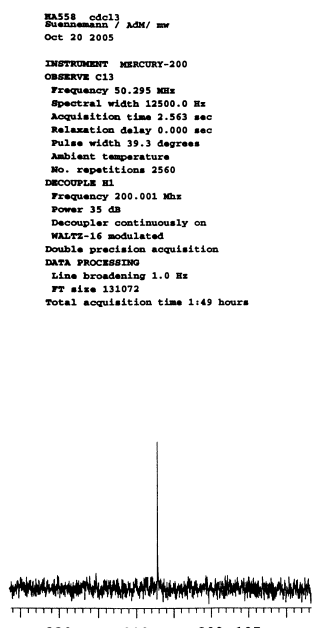

$220 \quad 210 \quad 200195$

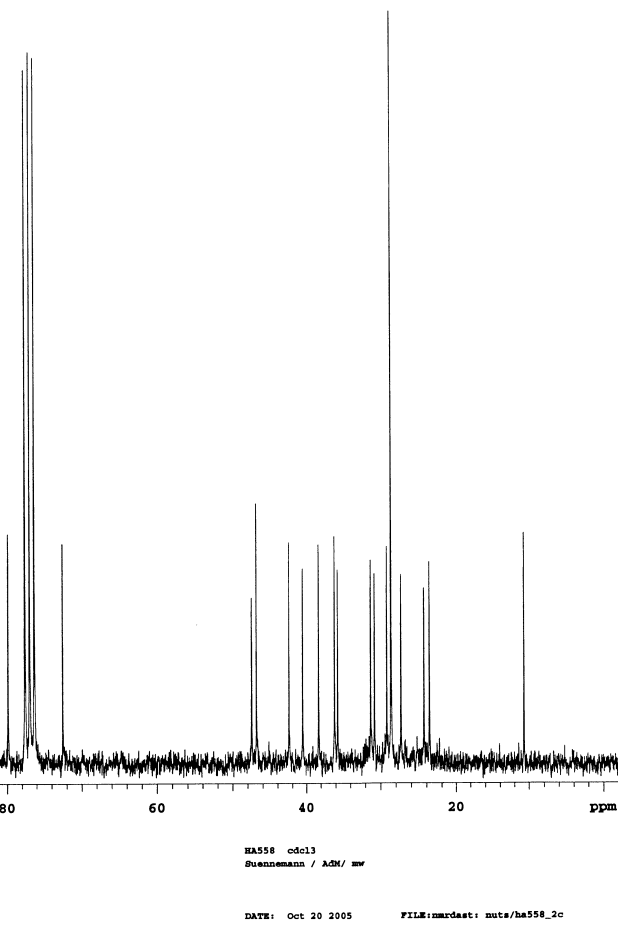



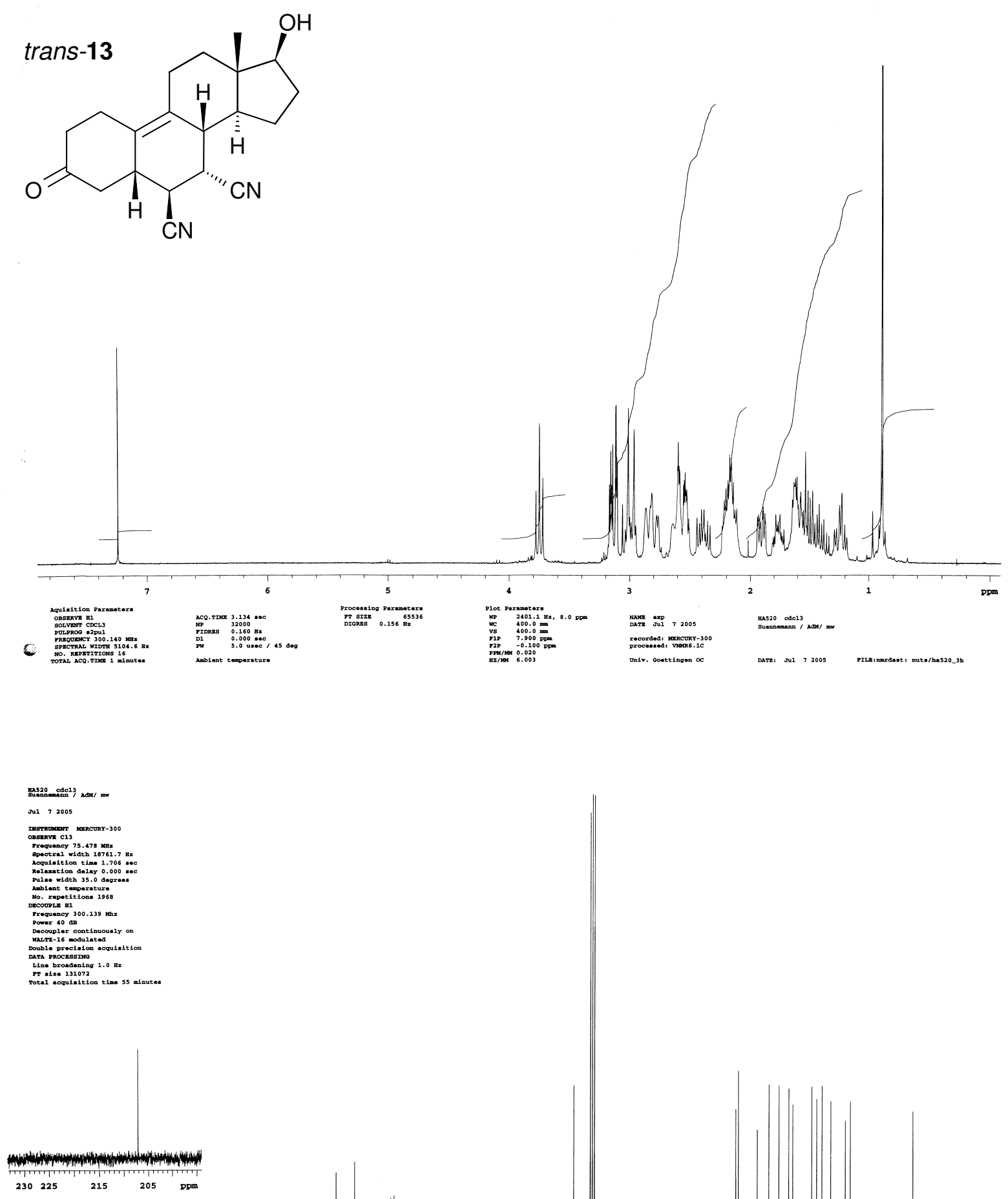

$\begin{array}{llll}230225 & 215 & 205 & 05\end{array}$

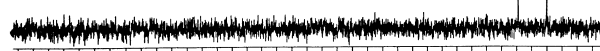



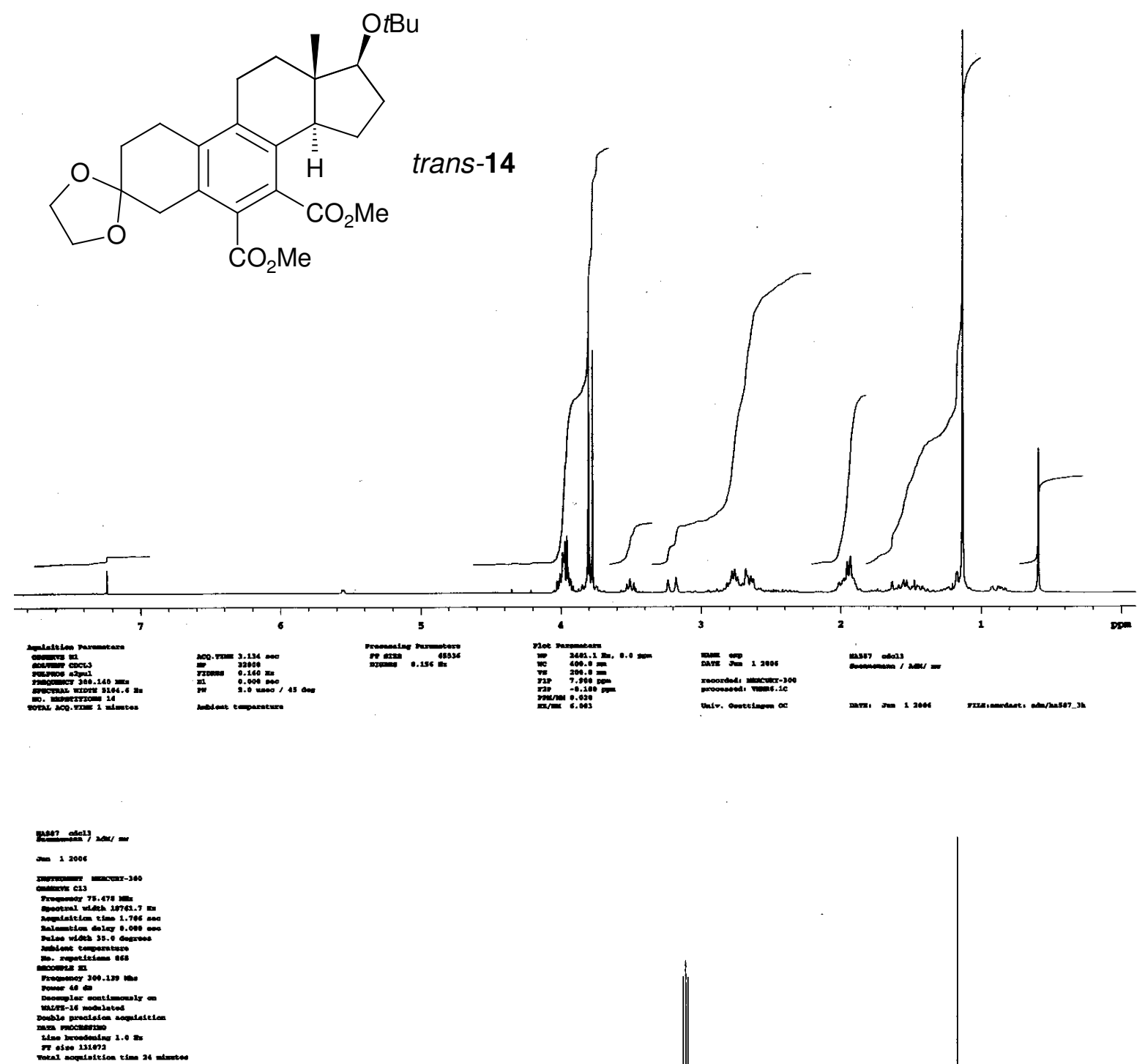

160

140

120

100

80

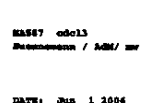

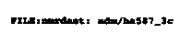


<smiles>CCCCC1CC=C2c3c(c(C(C)=O)c4c(c3C(C)=O)CC3(CC4)OCCO3)CC[C@@]21C</smiles>

15

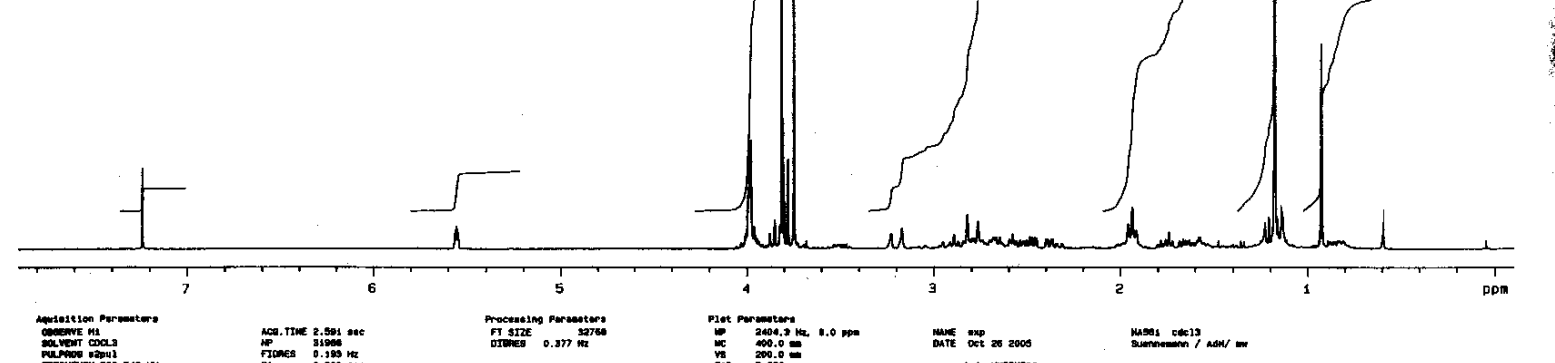

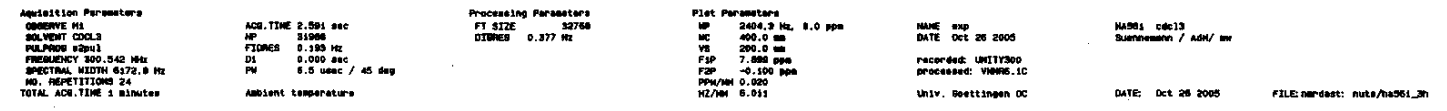

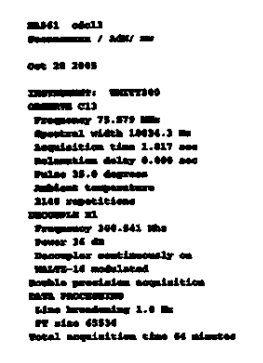

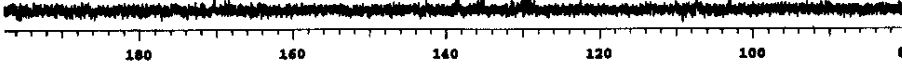

10
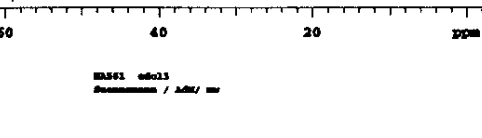


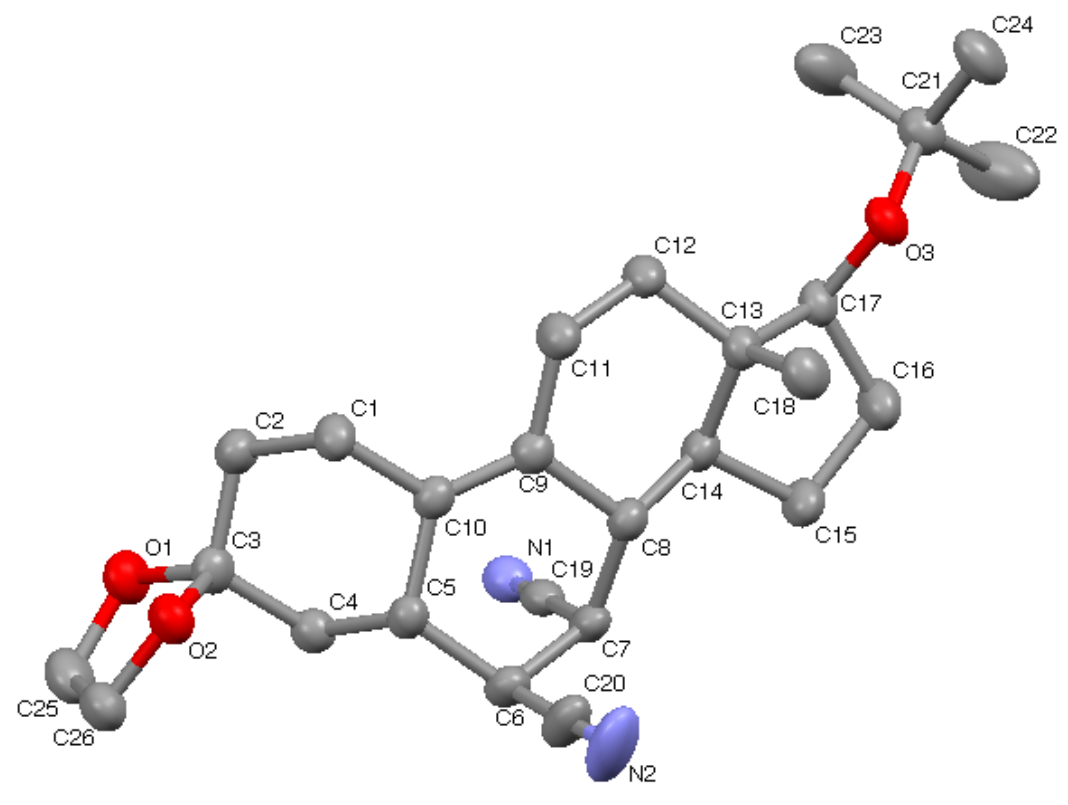

Figure S1. Molecular structure of $\mathrm{C}_{26} \mathrm{H}_{36} \mathrm{O}_{3} \mathrm{~N}_{2}$ (trans-5) with labeling of non-hydrogen atoms. Atomic displacement ellipsoids show 50\% probability levels. Hydrogen atoms have been omitted for clarity.

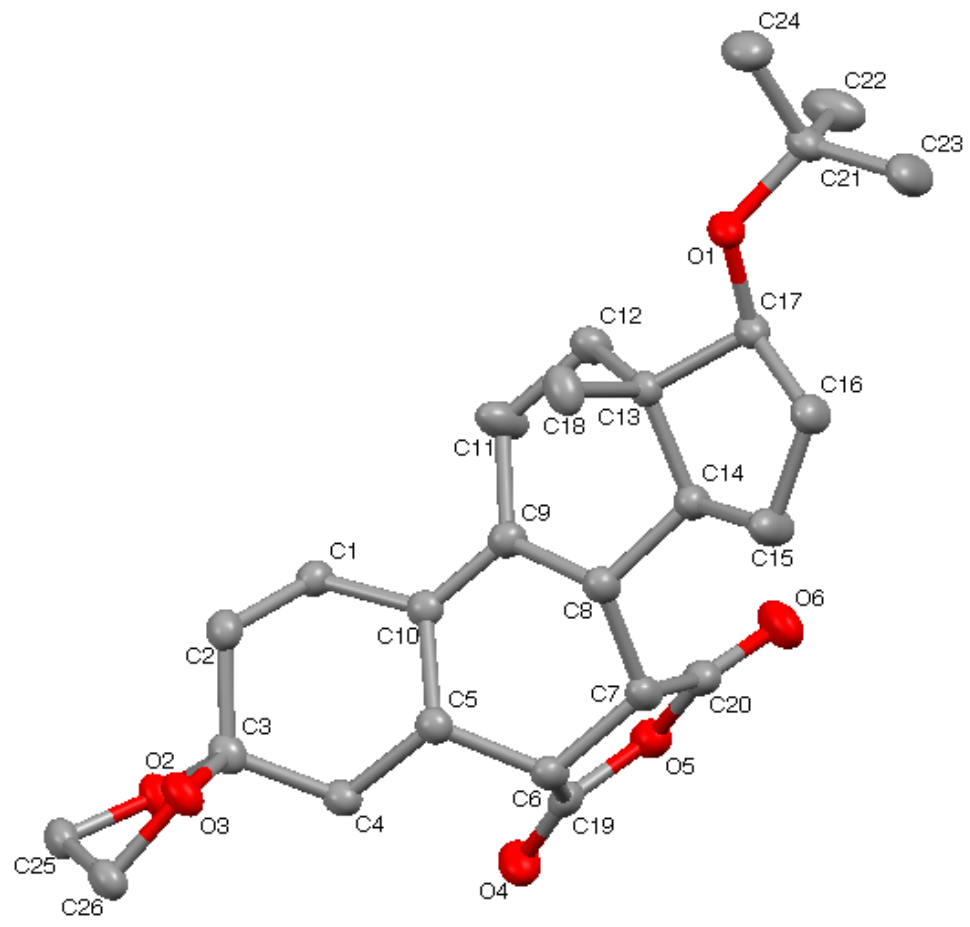

Figure S2. Molecular structure of $\mathrm{C}_{26} \mathrm{H}_{36} \mathrm{O}_{6}$ (trans-10) with labeling of non-hydrogen atoms. Atomic displacement ellipsoids show $50 \%$ probability levels. Hydrogen atoms have been omitted for clarity. 